\title{
Positron Scattering from Gas-Phase Beryllium and Magnesium: Theory, Recommended Cross Sections, and Transport Simulations
}

Cite as: J. Phys. Chem. Ref. Data 48, 033103 (2019); https://doi.org/10.1063/1.5115353

Submitted: 13 May 2019 . Accepted: 17 June 2019 . Published Online: 24 July 2019

F. Blanco (D), G. García (D) R. P. McEachran, P. W. Stokes, R. D. White, and M. J. Brunger (D)
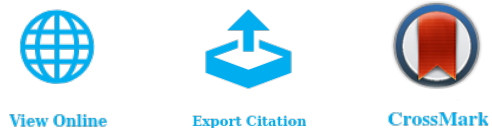

\section{ARTICLES YOU MAY BE INTERESTED IN}

EOS-LNG: A Fundamental Equation of State for the Calculation of Thermodynamic

Properties of Liquefied Natural Gases

Journal of Physical and Chemical Reference Data 48, 033102 (2019); https: //

doi.org/10.1063/1.5093800

Viscosity of Typical Room-Temperature Ionic Liquids: A Critical Review Journal of Physical and Chemical Reference Data 48, 033101 (2019); https:// doi.org/10.1063/1.5090486

Theoretical Energy Levels of 1sns and 1snp States of Helium-Like Ions Journal of Physical and Chemical Reference Data 48, 033104 (2019); https: // doi.org/10.1063/1.5121413

Journal of Physical and Chemical Reference Data SPECIAL TOPIC: 


\title{
Positron Scattering from Gas-Phase Beryllium and Magnesium: Theory, Recommended Cross Sections, and Transport Simulations
}

\author{
Cite as: J. Phys. Chem. Ref. Data 48, 033103 (2019); doi: 10.1063/1.5115353 \\ Submitted: 13 May 2019 • Accepted: 17 June 2019 • \\ Published Online: 24 July 2019
}

F. Blanco, ${ }^{7}$ (D) G. Garcia, ${ }^{2}$ (D) R. P. McEachran, ${ }^{3}$ P. W. Stokes, ${ }^{4}$ R. D. White, ${ }^{4}$ and M. J. Brunger ${ }^{5, a)}$ (D)

\begin{abstract}
AFFILIATIONS
${ }^{7}$ Departamento de Estructura de la Materia Física Térmica y Electrónica, e IPARCOS, Universidad Complutense de Madrid, Plaza de Ciencias 1, E-28040, Madrid, Spain

${ }^{2}$ Instituto de Física Fundamental, CSIC, Serrano 113-bis, E-28006, Madrid, Spain

${ }^{3}$ Plasma Research Laboratory, RSPE, Australian National University, Canberra, ACT 0200, Australia

${ }^{4}$ College of Science and Engineering, James Cook University, Townsville, Queensland 4810, Australia

${ }^{5}$ College of Science and Engineering, Flinders University, GPO Box 2100, Adelaide, SA 5001, Australia
\end{abstract}

a) Author to whom correspondence should be addressed: michael.brunger@flinders.edu.au

\begin{abstract}
Results from the application of our optical potential and relativistic optical potential models to positron scattering from gas-phase beryllium (Be) and magnesium $(\mathrm{Mg})$ are presented. Specifically, total cross sections and integral cross sections for the elastic, positronium formation, summed discrete electronic-state excitation, and ionization scattering processes are reported for both species and over an extended incident positron energy range. Where possible, these results are compared against the existing theoretical and experimental data, although it must be noted here that no current measurements are yet available for Be and those that are available for $\mathrm{Mg}$ are largely restricted to the total cross section. Nonetheless, on the basis of that comparison, recommended cross section datasets, for all the aforementioned cross sections, are formed. Those recommended cross section data are subsequently employed in a Boltzmann equation analysis to simulate the transport of positrons, under the influence of an applied (external) electric field, through the background Be and Mg gases. Note that relativistic optical potential results for the elastic momentum transfer cross section are also reported, to allow us to account for anisotropy effects in our transport simulations. Finally, our positron simulation results for quantities such as the ionization rate coefficients and flux and bulk drift velocities are compared with the corresponding electron transport results with significant differences being observed.
\end{abstract}

Published by AIP Publishing on behalf of the National Institute of Standards and Technology. https://doi.org/10.1063/1.5115353

Key words: beryllium; magnesium; positron scattering cross sections; positron transport; recommended cross sections.

\section{CONTENTS}

1. Introduction $\ldots \ldots \ldots \ldots \ldots \ldots \ldots \ldots \ldots \ldots \ldots \ldots \ldots \ldots \ldots \ldots \ldots \ldots \ldots$

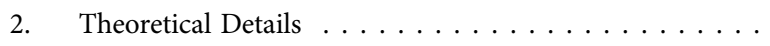

2.1. OP . . . . . . . . . . . . . . . .

2.2. ROP details $\ldots \ldots \ldots \ldots \ldots \ldots \ldots \ldots \ldots$

2.2.1. Positronium formation . . . . . . . . . . . .

2.2.2. The absorption potential . . . . . . . . .

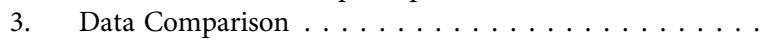

4. Recommended Data . . . . . . . . . . . . . . . .

5. Transport Simulations . . . . . . . . . . . . . .

5.1. Electron transport in $\mathrm{Be} \ldots \ldots \ldots \ldots \ldots \ldots$

5.2. Positron transport in gaseous $\mathrm{Be}$ and $\mathrm{Mg} \ldots \ldots$.
5.3. Comparison of electron and positron transport in gaseous $\mathrm{Be}$ and $\mathrm{Mg} \ldots \ldots \ldots \ldots \ldots$

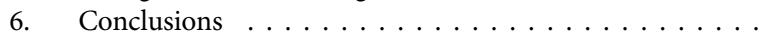
Acknowledgments . . . . . . . . . . . . . . . .

7. References ................. 15 18 19 19

\section{List of Tables}

1. The present theoretical OP results $\left(\times 10^{-16} \mathrm{~cm}^{2}\right)$ for positron scattering from beryllium . . . . . . . .

2. The present theoretical OP results $\left(\times 10^{-16} \mathrm{~cm}^{2}\right)$ for positron scattering from magnesium $\ldots \ldots \ldots \ldots$ 
3. A selection of the present ROP results $\left(\times 10^{-16} \mathrm{~cm}^{2}\right)$ for positron scattering from beryllium $\ldots \ldots \ldots \ldots \ldots$ A selection of the present ROP results $\left(\times 10^{-16} \mathrm{~cm}^{2}\right)$ for positron scattering from magnesium $\ldots \ldots \ldots \ldots$ . Recommended cross sections for positron scattering from Be . . . . . . . . . . . . . . . . Recommended cross sections for positron scattering from

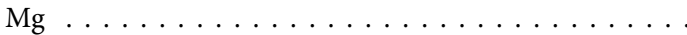

\section{List of Figures}

1. The present OP integral and total cross section results for positron scattering from beryllium . . . . . . . . .

2. The present $\mathrm{OP}$ integral and total cross section results for positron scattering from magnesium . . . . . . . . The present ROP integral and total cross section results for positron scattering from beryllium . . . . . . . . .

4. The present ROP integral and total cross section results for positron scattering from magnesium ......... Comparison of the present OP and ROP integral and total cross section results, against those from earlier studies, for positron-Be scattering $\ldots \ldots \ldots \ldots \ldots \ldots$
6. Comparison of the present $\mathrm{OP}$ and ROP integral and total cross section results, against those from earlier studies, for positron-Mg scattering . . . . . . . . . . . 7. Recommended integral and total cross sections for positron scattering from (a) Be and (b) $\mathrm{Mg} \ldots \ldots \ldots$. . . . 8. Comparison of the calculated rate coefficients for ionization (top), bulk (solid), and flux (dashed) drift velocities (bottom) for electrons in Be (blue) and $\mathrm{Mg}$ (orange) vapor at $750 \mathrm{~K}$ over a range of reduced electric fields ....................

9. Comparison of the calculated rate coefficients (top) for ionization (solid) and positronium formation (dashed), bulk (solid) and flux (dashed) drift velocities (bottom) for positrons in $\mathrm{Be}$ (blue) and $\mathrm{Mg}$ (orange) vapor at $750 \mathrm{~K}$ over a range of reduced electric fields . . . . . . . . .

10. Comparison of the calculated rate coefficients (top) for ionization (solid) and positronium formation (dashed), bulk (solid) and flux (dashed) drift velocities (bottom row) for electrons (blue) and positrons (orange) in $\mathrm{Be}$ (left column) and $\mathrm{Mg}$ (right column) vapor at $750 \mathrm{~K}$ over a range of reduced electric fields .................

\section{Introduction}

Previous investigations into positron scattering from beryllium $(\mathrm{Be})$ and magnesium $(\mathrm{Mg})$ have been limited in terms of either the types of processes considered (i.e., elastic, discrete inelastic, positronium formation, ionization, and the total cross section) and/or the energy range being studied. From an experimental perspective, the absence of a section on Be in the two recent positron-atom scattering reviews $^{1,2}$ is strong evidence for no such data existing in the literature. This situation, however, arises from the real practical challenges in working with Be and in trying to establish a source of gas-phase Beatoms. On the other hand, the situation is somewhat improved in terms of theoretical studies into this scattering system. In particular, we note the elastic integral cross section (ICS) results from Bromley et al., ${ }^{3}$ Mitroy and Ivanov, ${ }^{4}$ Reid and Wadehra, ${ }^{5}$ and Poveda et al. ${ }^{6}$ Note that below the positronium formation channel threshold energy $\left(E_{\mathrm{Ps}}=2.522 \mathrm{eV}\right)$ in $\mathrm{Be}$, those elastic ICSs are equivalent to the total scattering cross section (TCS). Further note that currently it appears that no theoretical results for the positronium formation, discrete inelastic, and ionization channels are available for positron-Be scattering. Rectifying this deficiency, through application of our optical potential (OP) and relativistic optical potential (ROP) computational methods, thus forms one rationale for this work. In regard to positron-Mg scattering, experimental data, largely from the group that was based at Wayne State University, can be found in the literature. ${ }^{7,8}$ Most of that data relates to the TCS, although a preliminary result for the positronium formation ICS was published in the work of Surdutovich et al. ${ }^{9}$ However, in their review, Ratnavelu et al. ${ }^{2}$ declined to use that preliminary data in order to form a recommended positronium formation cross section and as a consequence we do not consider it further. Similar to that just described above for Be, theoretical elastic ICSs for positron-Mg scattering are also available from Bromley et al., ${ }^{3}$ Mitroy and Ivanov, ${ }^{4}$ Reid and Wadehra, ${ }^{5}$ and Poveda et al. ${ }^{6}$ In this case, those elastic ICSs equate to the TCS below $0.846 \mathrm{eV}$, the positronium formation threshold energy of $\mathrm{Mg}$. In addition, there are also elastic ICS theoretical results from Mitroy et al., ${ }^{10}$ Savage et al. ${ }^{11}$ [a single-center convergent close coupling (CCC) result], and Utamuratov et al. ${ }^{12}$ (a two-center CCC result). Quite a significant degree of theoretical endeavor is also apparent in studying the positronium formation channel. This includes the results from Gribakin and King, ${ }^{13}$ Hewitt et al., ${ }^{14}$ Chang and Zhou, ${ }^{15}$ and Utamuratov et al. ${ }^{12}$ However, the agreement between those calculations for that channel is rather poor. Finally, we note that the only previous ionization ICS for the positron-Mg system is from Uramuratov et al., ${ }^{12}$ while there are no current results for the discrete inelastic channels. In order to try and better understand the discrepancies between the existing $\mathrm{Mg}$ calculations, and add to the database where only one or no computational result is currently available, we have also applied our OP and ROP methods to this scattering system. This forms another important rationale for the current investigation.

There has been quite a bit of historical interest in comparing electron and positron scattering cross section results, with significant experimental work in this respect being undertaken at Yamaguchi University (e.g., Refs. 16 and 17) and the University of Trento. ${ }^{18-20}$ The rationale, in part, behind those investigations was to see at what energy the electron and positron results (usually at the TCS level) converged to the same values. Typically, that convergence was thought to occur once the exchange interaction (in electron scattering) and positronium formation (in positron scattering) became so small that they could be neglected. At that point, the positron and electron interactions, from a given target, were considered to be largely identical so that their cross sections converged. This was found to be the case, to within the uncertainties on the data, in many examples, ${ }^{21}$ although the energy at which the cross section values merged was very target specific. ${ }^{16-20}$ Note that while the polarization interaction in both the electron and positron scattering cases does act 
the same, due to the different charges the static interaction, in principle, remains different at all energies. We therefore also wished to investigate this effect with our OP and ROP calculations in both $\mathrm{Be}$ and $\mathrm{Mg}$, drawing on our recent electron- $\mathrm{Be}^{22}$ and electron- $\mathrm{Mg}^{23}$ results in order to do so. Furthermore, and we believe this is the first time such a study has been undertaken, we intend to use our cross section data for electron and positron scattering in order to simulate their transport characteristics through background Be and Mg gases, under the influence of an applied (external) electric field, and to investigate any similarities or differences in their behavior across a range of reduced electric fields $\left(E / n_{0}\right.$, where $E=$ electric field strength and $n_{0}=$ number density).

The structure of the remainder of this paper is as follows: In Sec. 2, we provide details of our OP and ROP calculations, while in Sec. 3 these results are compared and discussed against those, for both $\mathrm{Be}$ and $\mathrm{Mg}$, which are currently available in the literature.
On the basis of that comparison, in Sec. 4, we formulate the recommended data for those species for total scattering, elastic scattering, positronium formation, the sum of all the discrete inelastic channels, and ionization. Using these recommended positron cross section sets, and corresponding data (where appropriate) for electron scattering, ${ }^{22,23}$ in Sec. 5, the results from our Boltzmann equation analysis of positron and electron transport in $\mathrm{Be}$ and $\mathrm{Mg}$ are presented. Brief details of our simulation methodology are also given in Sec. 5. Finally, in Sec. 6, some conclusions from this investigation are outlined.

\section{Theoretical Details}

In this section, we provide some brief details with respect to our current OP and ROP theoretical methods and their application to positron $\left(e^{+}\right)$scattering processes.

TABLE 1. The present theoretical OP results $\left(\times 10^{-16} \mathrm{~cm}^{2}\right)$ for positron scattering from beryllium

\begin{tabular}{|c|c|c|c|c|c|}
\hline Energy $(\mathrm{eV})$ & $\begin{array}{c}\text { Elastic ICS } \\
\left(\times 10^{-16} \mathrm{~cm}^{2}\right)\end{array}$ & $\begin{array}{l}\text { Ps formation } \\
\left(\times 10^{-16} \mathrm{~cm}^{2}\right)\end{array}$ & $\begin{array}{c}\text { Excitation } \\
\left(\times 10^{-16} \mathrm{~cm}^{2}\right)\end{array}$ & $\begin{array}{l}\text { Direct ionization } \\
\text { ICS }\left(\times 10^{-16} \mathrm{~cm}^{2}\right)\end{array}$ & $\begin{array}{c}\text { Total } \\
\left(\times 10^{-16} \mathrm{~cm}^{2}\right)\end{array}$ \\
\hline 0.1 & 383.6 & 0 & 0 & 0 & 383.6 \\
\hline 0.15 & 296.8 & 0 & 0 & 0 & 296.8 \\
\hline 0.2 & 242.76 & 0 & 0 & 0 & 242.76 \\
\hline 0.3 & 177.8 & 0 & 0 & 0 & 177.8 \\
\hline 0.4 & 141.96 & 0 & 0 & 0 & 141.96 \\
\hline 0.5 & 119.84 & 0 & 0 & 0 & 119.84 \\
\hline 0.7 & 93.8 & 0 & 0 & 0 & 93.8 \\
\hline 1 & 72.8 & 0 & 0 & 0 & 72.8 \\
\hline 1.5 & 53.48 & 0 & 0 & 0 & 53.48 \\
\hline 2 & 42 & 0 & 0 & 0 & 42 \\
\hline 3 & 28.84 & 0 & 0 & 0 & 28.84 \\
\hline 4 & 21.952 & 0.5208 & 0 & 0 & 22.4728 \\
\hline 5 & 15.904 & 6.636 & 0 & 0 & 22.54 \\
\hline 7 & 10.92 & 11.284 & 3.556 & 0 & 25.76 \\
\hline 10 & 9.716 & 7.084 & 9.06808 & 0.14392 & 26.012 \\
\hline 15 & 8.708 & 4.704 & 6.944 & 3.5 & 23.856 \\
\hline 20 & 7.896 & 4.508 & 4.844 & 4.872 & 22.12 \\
\hline 30 & 6.468 & 4.284 & 3.388 & 4.76 & 18.9 \\
\hline 40 & 4.844 & 3.108 & 2.772 & 4.228 & 14.952 \\
\hline 50 & 3.612 & 1.904 & 2.408 & 3.752 & 11.676 \\
\hline 70 & 2.408 & 0.728 & 1.96 & 3.052 & 8.148 \\
\hline 100 & 1.68 & 0.224 & 1.554 & 2.422 & 5.88 \\
\hline 150 & 1.1564 & 0.056 & 1.1732 & 1.8228 & 4.2084 \\
\hline 200 & 0.8932 & 0.028 & 0.9436 & 1.4784 & 3.3432 \\
\hline 300 & 0.6272 & 0 & 0.6888 & 1.0864 & 2.4108 \\
\hline 400 & 0.4844 & 0 & 0.546 & 0.8652 & 1.8984 \\
\hline 500 & 0.3976 & 0 & 0.4564 & 0.7252 & 1.582 \\
\hline 700 & 0.2912 & 0 & 0.3472 & 0.5488 & 1.1872 \\
\hline 1000 & 0.20944 & 0 & 0.2576 & 0.4088 & 0.87584 \\
\hline 2000 & 0.10612 & 0 & 0.14672 & 0.22288 & 0.47572 \\
\hline 3000 & 0.07028 & 0 & 0.10276 & 0.15372 & 0.32676 \\
\hline 4000 & 0.05292 & 0 & 0.08036 & 0.11816 & 0.25144 \\
\hline 5000 & 0.04256 & 0 & 0.06608 & 0.09576 & 0.2044 \\
\hline 7000 & 0.03108 & 0 & 0.049 & 0.06916 & 0.14924 \\
\hline 10000 & 0.023212 & 0 & 0.03528 & 0.049 & 0.107492 \\
\hline
\end{tabular}




\subsection{OP}

Our OP method is based on a local complex potential representing the atomic scattering center, according to the following equation:

$$
V(r)=V_{s}(r)+V_{p}(r)-i V_{a}(r),
$$

where $V_{s}(r)+V_{p}(r)$ is the real or elastic scattering part of the OP and $i V_{a}(r)$ is the imaginary absorption potential, accounting for the inelastic scattering channels. $V_{s}(r)$ represents the static potential, describing the interaction between the positron and the atomic charge density, and is repulsive in the case of positrons. We formulate this on a derivation of the Hartree-Fock atomic wavefunctions, analogous to the work of Reid and Wadehra. ${ }^{24} V_{p}(r)$ represents the polarization potential which accounts for the target electron cloud deformation during the collision and is therefore dependent on its atomic polarizability. We have shown in previous studies with oxygen $(\mathrm{O})$ containing molecular targets how sensitive low energy elastic positron scattering is to the accuracy on the description of this term. ${ }^{25,26}$ In that case, we used the sum of a dipole and quadrupole potential calculated with the polarized-orbital method, by determining the first-order corrections of the atomic orbitals due to a fixed charge field. ${ }^{27}$ For O, the dipole and quadrupole polarized orbital potential of $\mathrm{Ne}$ (accurate against measurement ${ }^{28}$ ) was scaled according to the procedure described in the work of Chiari et al. ${ }^{26}$ A similar procedure is not currently available for Be and $\mathrm{Mg}$ so we decided to use the energy dependent polarization potential quite recently proposed by Reid and Wadehra. ${ }^{5} V_{a}(r)$ describes the "absorption" processes, i.e., the inelastic processes of excitation, ionization, and positronium formation (Ps), and require careful treatment. We use a scheme modified from that proposed by Reid and Wadehra, ${ }^{24}$ assuming that the target electrons can be considered as a quasifree electron cloud with which the incoming particles undergo binary collisions. The threshold energy is carefully designed to include Ps formation.

Our recent improvements to the treatment of Ps formation are outlined in detail previously. ${ }^{29}$ In brief, we use an energy dependent threshold $\Delta(E)$ coinciding with the well-known Ps formation threshold of $\Delta_{p}=I-6.8 \mathrm{eV}$ (where $I=$ ionization threshold), for lower energies, and the lowest optically allowed excitation transition $\Delta$ for higher impact energies by assuming a smooth transition in threshold energy, from low to high impact energy, which follows the expression

$$
\Delta(E)=\Delta-\frac{\left(\Delta-\Delta_{p}\right)}{\left[1+\left(\frac{E}{3 I}-1\right)^{2}\right]} .
$$

Hence, we used $\Delta=5.28 \mathrm{eV}$ for $\mathrm{Be}$, but in the particular case of $\mathrm{Mg}$ instead of the lowest optically allowed excitation transition we employed $\Delta=2.71 \mathrm{eV}$, which corresponds to a forbidden transition which, probably due to the strong configuration mixing present in $\mathrm{Mg}$, contributes notably to the emission spectrum. ${ }^{23}$

The present $\mathrm{OP}$ results for positron scattering from $\mathrm{Be}$ are summarized in Table 1 and Fig. 1, while those for positron scattering from $\mathrm{Mg}$ are summarized in Table 2 and Fig. 2.

\subsection{ROP details}

The theoretical procedures used here to describe the elastic and inelastic scattering of positrons from beryllium and magnesium

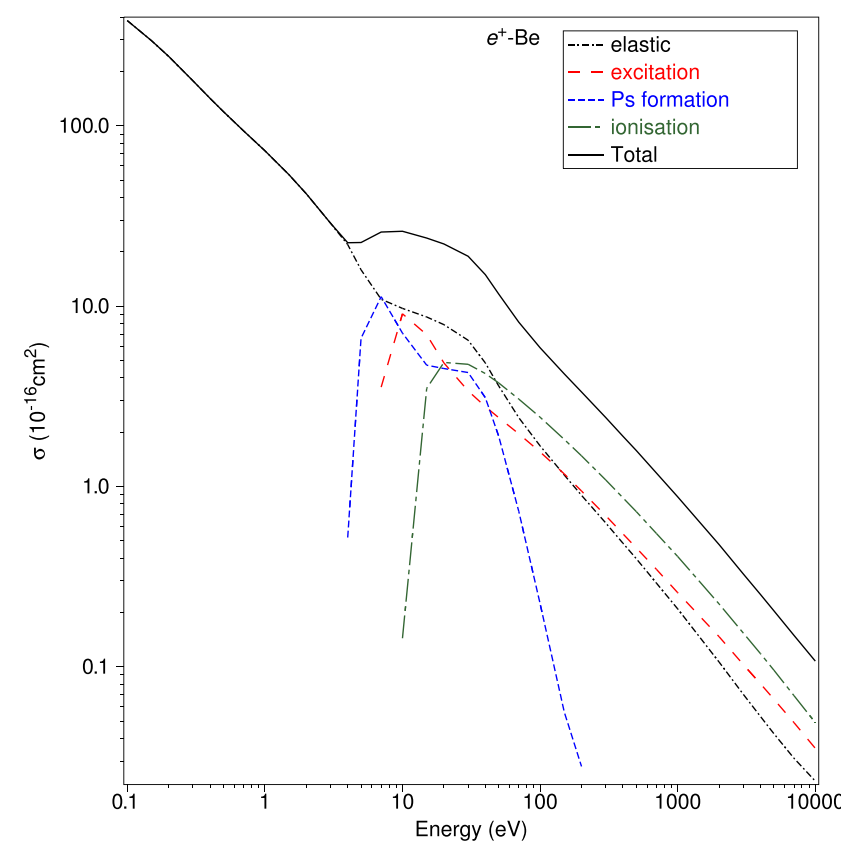

FIG. 1. The present $\mathrm{OP}$ integral and total cross section results for positron scattering from beryllium. See the legend in the figure for the processes considered.

atoms is based upon the ROP method of Chen et al., ${ }^{30}$ hereafter referred to as Paper I. This OP method is based upon an approximate solution of the relativistic close-coupling equations. Only a brief discussion of the overall method will be given here, and the reader is referred to Ref. I for details.

The scattering of the incident positrons, with wavenumber $k$, by beryllium and magnesium atoms is described by the integral equation formulation of the partial wave Dirac-Fock scattering equations. In the ROP method, these equations can be expressed in matrix form as

$$
\begin{aligned}
\left(\begin{array}{l}
F_{\mathrm{\kappa}}(r) \\
G_{\mathrm{\kappa}}(r)
\end{array}\right)= & \left(\begin{array}{l}
v_{1}(k r) \\
v_{2}(k r)
\end{array}\right)+\frac{1}{k} \int_{0}^{r} \mathrm{~d} x G(x, r) \\
& \times\left[U(r)\left(\begin{array}{c}
F_{\mathrm{\kappa}}(r) \\
G_{\mathrm{\kappa}}(r)
\end{array}\right)-i U_{\mathrm{a}}(r)\left(\begin{array}{c}
F_{\mathrm{\kappa}}(r) \\
G_{\mathrm{\kappa}}(r)
\end{array}\right)\right],
\end{aligned}
$$

where the local potential $U(r)$ is given by the sum of the static and local polarization potentials, i.e.,

$$
U(r)=U_{\text {st }}(r)+U_{\text {pol }}(r) .
$$

Here, we used the procedure outlined by Bartschat et al., ${ }^{31,32}$ whereby the real part of the OP was replaced by a local polarization potential based upon a polarized-orbital method of McEachran et al. ${ }^{27,33}$ The static potentials were determined in the usual manner from the DiracFock orbitals of the atoms. The polarization potentials $U_{\text {pol }}(r)$ in Eq. (4) comprised the sum of the first 11 multipole polarization potentials using the polarized-orbital method of McEachran et al. ${ }^{27}$ This potential was then augmented by an additional potential obtained by an extrapolation to an infinite number of multipoles as detailed in the work of McEachran et al. ${ }^{33}$ In Eq. (3), the nonlocal potential $U_{\mathrm{a}}(r)$ denotes the imaginary part of the OP and, in turn, describes the 
TABLE 2. The present theoretical OP results $\left(\times 10^{-16} \mathrm{~cm}^{2}\right)$ for positron scattering from magnesium

\begin{tabular}{|c|c|c|c|c|c|}
\hline Energy $(\mathrm{eV})$ & $\begin{array}{c}\text { Elastic ICS } \\
\left(\times 10^{-16} \mathrm{~cm}^{2}\right)\end{array}$ & $\begin{array}{l}\text { Ps formation } \\
\left(\times 10^{-16} \mathrm{~cm}^{2}\right)\end{array}$ & $\begin{array}{c}\text { Excitation } \\
\left(\times 10^{-16} \mathrm{~cm}^{2}\right)\end{array}$ & $\begin{array}{l}\text { Direct ionization } \\
\text { ICS }\left(\times 10^{-16} \mathrm{~cm}^{2}\right)\end{array}$ & $\begin{array}{c}\text { Total } \\
\left(\times 10^{-16} \mathrm{~cm}^{2}\right)\end{array}$ \\
\hline 0.1 & 226.52 & 0 & 0 & 0 & 226.52 \\
\hline 0.15 & 237.16 & 0 & 0 & 0 & 237.16 \\
\hline 0.2 & 249.76 & 0 & 0 & 0 & 249.76 \\
\hline 0.3 & 268.52 & 0 & 0 & 0 & 268.52 \\
\hline 0.4 & 271.04 & 0 & 0 & 0 & 271.04 \\
\hline 0.5 & 260.12 & 0 & 0 & 0 & 260.12 \\
\hline 0.7 & 222.6 & 0 & 0 & 0 & 222.6 \\
\hline 1 & 171.92 & 0 & 0 & 0 & 171.92 \\
\hline 1.5 & 120.96 & 0 & 0 & 0 & 120.96 \\
\hline 2 & 86.52 & 7.448 & 0 & 0 & 93.968 \\
\hline 3 & 35.84 & 35.042 & 1.638 & 0 & 72.52 \\
\hline 4 & 26.516 & 20.16 & 16.8 & 0 & 63.476 \\
\hline 5 & 23.296 & 10.388 & 24.332 & 0 & 58.016 \\
\hline 7 & 20.412 & 6.16 & 25.76 & 0 & 52.332 \\
\hline 10 & 18.648 & 6.244 & 20.496 & 2.94 & 48.328 \\
\hline 15 & 17.276 & 7.476 & 12.516 & 7.812 & 45.08 \\
\hline 20 & 16.436 & 8.596 & 9.912 & 8.232 & 43.176 \\
\hline 30 & 13.272 & 7.336 & 7.84 & 7.336 & 35.784 \\
\hline 40 & 9.8 & 4.116 & 6.776 & 6.44 & 27.132 \\
\hline 50 & 7.728 & 2.268 & 6.02 & 5.768 & 21.784 \\
\hline 70 & 5.656 & 0.812 & 5.04 & 4.788 & 16.296 \\
\hline 100 & 4.228 & 0.28 & 4.088 & 3.892 & 12.488 \\
\hline 150 & 3.08 & 0.084 & 3.164 & 3.052 & 9.38 \\
\hline 200 & 2.4668 & 0.056 & 2.6096 & 2.5424 & 7.6748 \\
\hline 300 & 1.8144 & 0 & 1.9852 & 1.9628 & 5.7624 \\
\hline 400 & 1.456 & 0 & 1.6212 & 1.6268 & 4.704 \\
\hline 500 & 1.2376 & 0 & 1.386 & 1.414 & 4.0376 \\
\hline 700 & 0.9576 & 0 & 1.0976 & 1.1312 & 3.1892 \\
\hline 1000 & 0.7308 & 0 & 0.8652 & 0.8932 & 2.492 \\
\hline 2000 & 0.42 & 0 & 0.5628 & 0.5516 & 1.5344 \\
\hline 3000 & 0.2968 & 0 & 0.4396 & 0.4088 & 1.1452 \\
\hline 4000 & 0.22988 & 0 & 0.3724 & 0.3276 & 0.92988 \\
\hline 5000 & 0.18732 & 0 & 0.32452 & 0.27468 & 0.78652 \\
\hline 7000 & 0.13636 & 0 & 0.26152 & 0.20888 & 0.60676 \\
\hline 10000 & 0.09688 & 0 & 0.20664 & 0.15456 & 0.45808 \\
\hline
\end{tabular}

absorption of the incident flux into the inelastic channels and thereby describes both excitation and ionization processes. This potential is given by a sum and integration over the bound and continuum states of the atom [see Sec. 2.2.1 as well as Eq. (21b) of Ref. I for details].

Finally, in Eq. (3), $F_{\kappa}(r)$ and $G_{\kappa}(r)$ are the large and small components of the complex scattering wavefunctions, while the functions $v_{1}(k r)$ and $v_{2}(k r)$ are the corresponding free particle wavefunctions and are given in terms of Riccati-Bessel functions. $G(r, x)$ is the free particle Green's function which can be expressed in matrix form in terms of the Riccati-Bessel and Riccati-Neumann functions [see Eqs. (23) and (24a,b) of Ref. I for details]. The subscript $\kappa$ on the scattering wavefunctions is the relativistic angular momentum quantum number of the incident positron. It is related to the corresponding orbital angular momentum quantum number $l$ according to $\kappa=-l-1$ when $j=l+\frac{1}{2}$ (spin-up) and $\kappa=l$ when $j=l-\frac{1}{2}$ (spin-down), where $j$ is the total angular momentum quantum number of the incident positron.

The large component of the complex scattering wavefunction is given asymptotically by

$$
F_{\kappa}(r)_{r \rightarrow \infty} \longrightarrow \sin \left(k r-\frac{l \pi}{2}\right)+T_{l}^{ \pm}(k) \exp \left(i k r-\frac{l \pi}{2}\right),
$$

where $T_{l}^{ \pm}(k)$ are the complex $T$-matrix elements. These $T$-matrix elements can be expressed in terms of the complex phase shifts $\eta_{l}^{ \pm}(k)$ according to

$$
T_{l}^{ \pm}(k)=\frac{1}{2 i}\left[\exp \left(2 i \eta_{l}^{ \pm}(k)\right)-1\right],
$$

where the real and imaginary parts of the phase shifts are given by

$$
\eta_{l}^{ \pm}(k)=\delta_{l}^{ \pm}(k)+i \gamma_{l}^{ \pm}(k)
$$




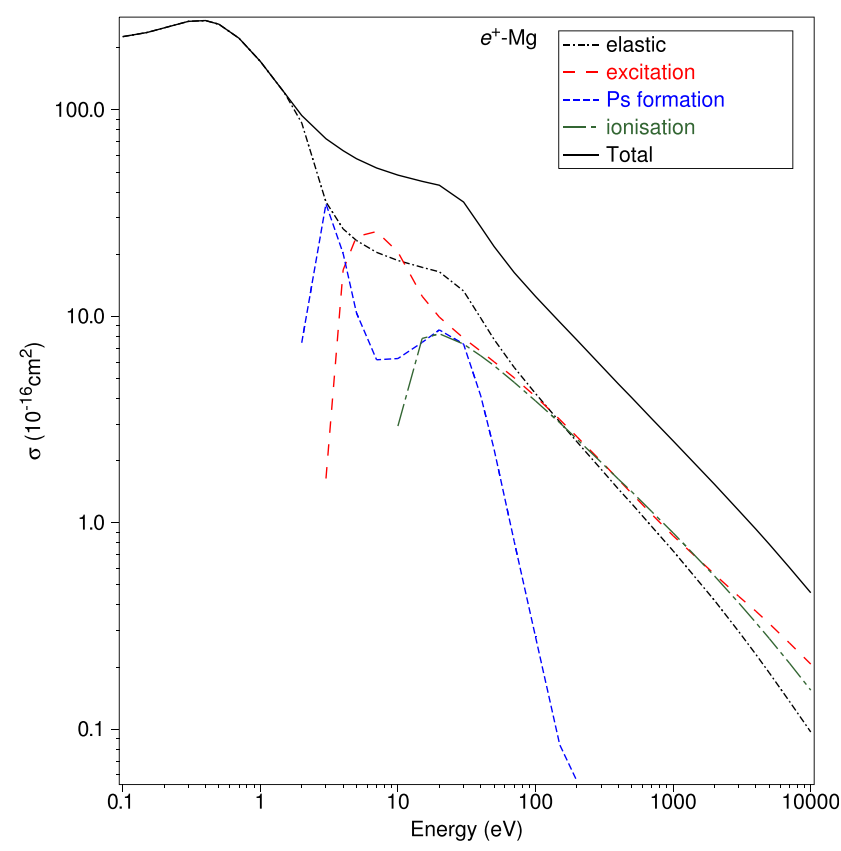

FIG. 2. The present OP integral and total cross section results for positron scattering from magnesium. See the legend in the figure for the processes considered.

Here, the superscript + refers to "spin-up," while the superscript refers to "spin-down." The integrated elastic cross section is then given, in terms of these real and imaginary parts of the phase shifts, by

$$
\begin{aligned}
\sigma^{\mathrm{el}}\left(k^{2}\right)= & \frac{2 \pi}{k^{2}} \sum_{l=0}^{\infty}\left\{(l+1) \exp \left(-2 \gamma_{l}^{+}\right)\left[\cosh 2 \gamma_{l}^{+}-\cos 2 \delta_{l}^{+}\right]\right. \\
& \left.+l \exp \left(-2 \gamma_{l}^{-}\right)\left[\cosh 2 \gamma_{l}^{-}-\cos 2 \delta_{l}^{-}\right]\right\},
\end{aligned}
$$

while the total inelastic or absorption cross section is given by

$$
\sigma^{\text {inel }}\left(k^{2}\right)=\frac{\pi}{k^{2}} \sum_{l=0}^{\infty}\left\{(l+1)\left[1-\exp \left(-4 \gamma_{l}^{+}\right)\right]+l\left[1-\exp \left(-4 \gamma_{l}^{-}\right)\right]\right\} \text {. }
$$

\subsubsection{Positronium formation}

Here, positronium formation was simulated using the method given in the work of McEachran and Stauffer ${ }^{34}$ which, in turn, is a modification of that originally suggested by Reid and Wadehra. ${ }^{24,35}$ In this method, the Ps formation cross section is calculated by first determining the direct ionization cross section and then the comparable cross section when the ionization thresholds are reduced by $6.8 \mathrm{eV}$, the binding energy of the ground state of positronium. The Ps formation cross section is then taken to be the difference between these two cross sections. Similarly, Ps formation in an excited state with principle quantum number $n$ can be simulated by reducing the ionization thresholds by $6.8 / n^{2} \mathrm{eV}$. Here, we included the $n=2,3$, and 4 excited states of positronium.

Any method for simulating rather than directly calculating Ps formation will contain one or more adjustable parameters. In the method of McEachran and Stauffer, ${ }^{34}$ there is just one adjustable parameter which is chosen according to where the Ps formation cross section effectively vanishes. For the noble gases, for which there are many experimental measurements, this parameter was chosen to be $120+E_{\text {ion }} \mathrm{eV}$. For two-electron systems, there are hardly any experimental measurements of Ps formation, except for helium. Consequently, based upon the measurements of Murtagh et al., ${ }^{36}$ the cutoff here was chosen to be $200+E_{\text {ion }} \mathrm{eV}$. It should be noted that in the method of McEachran and Stauffer, ${ }^{34}$ this adjustable parameter influences the asymptotic behavior of the Ps formation cross section but has very little influence on its peak value.

\subsubsection{The absorption potential}

For beryllium, the following 10 bound excited states were included in $U_{\mathrm{a}}(r)$, namely, $n \mathrm{p}^{1,3} \mathrm{P}^{\mathrm{o}}$ with $n=2-6$ in order to simulate excitation processes. Similarly, for magnesium, the 10 bound excited states included in $U_{\mathrm{a}}(r)$ were $n \mathrm{p}^{1,3} \mathrm{P}^{\mathrm{o}}$ with $n=3-7$. Also included in $U_{\mathrm{a}}(r)$, for both beryllium and magnesium, were all continuum states with the orbital angular momentum given by $l_{c}=0,1,2,3$, and 4 in order to simulate ionization processes. The integration over the continuum states in the absorption potential was approximated by using Gauss-Legendre integration with $16-20$ points. In a relativistic close-coupling expansion, it is necessary to couple the total angular momentum of the electron in the excited state (bound or continuum) to the total angular momentum of the incident positron in order to obtain the total angular momentum $J$ of the positron-atom system. This total angular momentum $J$ is then conserved during the collision process. Under the above circumstances, this gave rise to a maximum of 30 excitation channels and 84 ionization channels in $U_{\mathrm{a}}(r)$ for both beryllium and magnesium.

The present ROP results for positron scattering from Be are summarized in Table 3 and Fig. 3, while those for positron scattering from $\mathrm{Mg}$ are summarized in Table 4 and Fig. 4.

\section{Data Comparison}

In Fig. 5, we plot the available data ${ }^{3-6}$ for positron scattering from $\mathrm{Be}$, along with our OP and ROP results, for elastic scattering, positronium formation, the sum over discrete electronic-state excitation, ionization, and total scattering. There are several general observations we can make in relation to Fig. 5. First, as noted earlier, there are no experimental results against which we can compare the data from the theoretical computations. In addition, for positronium formation, discrete electronic-state excitation, and ionization only, the present OP and ROP results are now available. Considering first the elastic ICS at very low energies, then with the exception of our ROP calculation, which is too high in magnitude, the results from Poveda et al., ${ }^{6}$ Bromley et al., ${ }^{3}$ Mitroy and Ivanov, ${ }^{4}$ and Reid and Wadehra $^{5}$ are all in quite good accord. Note that in the work of McEachran et al., ${ }^{22,23}$ we had previously described that the ROP method at lower energies was not expected to be as accurate as close coupling results, and this point is reinforced in Fig. 5(a) although now for positron scattering. At energies above about $1 \mathrm{eV}$, all the available theoretical results, in their energy regions of overlap, are in very good agreement. In particular, we highlight the excellent accord between our OP and ROP results up to $5000 \mathrm{eV}$. For the positronium formation [Fig. 5(b)], sum over discrete electronic-state excitation [Fig. 5(c)], and ionization [Fig. 5(d)], our OP and ROP calculations 
TABLE 3. A selection of the present ROP results $\left(\times 10^{-16} \mathrm{~cm}^{2}\right)$ for positron scattering from beryllium. Note that the acronym MTCS denotes the momentum transfer cross section, used in Sec. 5 in the transport simulations (see later)

\begin{tabular}{|c|c|c|c|c|c|c|}
\hline Energy (eV) & $\begin{array}{c}\text { Elastic ICS } \\
\left(\times 10^{-16} \mathrm{~cm}^{2}\right)\end{array}$ & $\begin{array}{c}\text { MTCS } \\
\left(\times 10^{-16} \mathrm{~cm}^{2}\right)\end{array}$ & $\begin{array}{c}\text { Excitation } \\
\left(\times 10^{-16} \mathrm{~cm}^{2}\right)\end{array}$ & $\begin{array}{l}\text { Direct ionization } \\
\text { ICS }\left(\times 10^{-16} \mathrm{~cm}^{2}\right)\end{array}$ & $\begin{array}{l}\text { Ps formation } \\
\left(\times 10^{-16} \mathrm{~cm}^{2}\right)\end{array}$ & $\begin{array}{c}\text { Total } \\
\left(\times 10^{-16} \mathrm{~cm}^{2}\right)\end{array}$ \\
\hline 0.0000 & 3514.0300 & 3514.0300 & 0.0000 & 0.0000 & 0.0000 & 3514.0300 \\
\hline 0.0100 & 2268.8500 & 2297.7600 & 0.0000 & 0.0000 & 0.0000 & 2268.8500 \\
\hline 0.0200 & 1650.8800 & 1677.6800 & 0.0000 & 0.0000 & 0.0000 & 1650.8800 \\
\hline 0.0400 & 1054.0500 & 1072.1100 & 0.0000 & 0.0000 & 0.0000 & 1054.0500 \\
\hline 0.0600 & 764.9700 & 774.9200 & 0.0000 & 0.0000 & 0.0000 & 764.9700 \\
\hline 0.0800 & 595.3500 & 598.4400 & 0.0000 & 0.0000 & 0.0000 & 595.3500 \\
\hline 0.1200 & 406.5100 & 398.6800 & 0.0000 & 0.0000 & 0.0000 & 406.5100 \\
\hline 0.1600 & 305.1700 & 289.0000 & 0.0000 & 0.0000 & 0.0000 & 305.1700 \\
\hline 0.2500 & 192.9800 & 163.8500 & 0.0000 & 0.0000 & 0.0000 & 192.9800 \\
\hline 0.3500 & 137.3500 & 99.8200 & 0.0000 & 0.0000 & 0.0000 & 137.3500 \\
\hline 0.4500 & 108.0800 & 66.1500 & 0.0000 & 0.0000 & 0.0000 & 108.0800 \\
\hline 0.5500 & 90.4100 & 46.7000 & 0.0000 & 0.0000 & 0.0000 & 90.4100 \\
\hline 0.6570 & 77.9100 & 34.1600 & 0.0000 & 0.0000 & 0.0000 & 77.9100 \\
\hline 0.9000 & 60.6700 & 20.3800 & 0.0000 & 0.0000 & 0.0000 & 60.6700 \\
\hline 1.2000 & 48.2400 & 13.9800 & 0.0000 & 0.0000 & 0.0000 & 48.2400 \\
\hline 1.8000 & 34.0900 & 9.4300 & 0.0000 & 0.0000 & 0.0000 & 34.0900 \\
\hline 2.6000 & 24.4086 & 6.7824 & 0.0000 & 0.0000 & 0.1183 & 24.5269 \\
\hline 2.7000 & 23.5449 & 6.5248 & 0.0000 & 0.0000 & 0.3710 & 23.9159 \\
\hline 2.8000 & 22.7218 & 6.2754 & 0.0164 & 0.0000 & 0.6707 & 23.4089 \\
\hline 2.9000 & 21.9538 & 6.0409 & 0.0237 & 0.0000 & 0.9898 & 22.9673 \\
\hline 3.0000 & 21.2345 & 5.8202 & 0.0281 & 0.0000 & 1.3140 & 22.5766 \\
\hline 3.4000 & 18.7751 & 5.0627 & 0.0359 & 0.0000 & 2.5381 & 21.3491 \\
\hline 4.0000 & 16.0382 & 4.2179 & 0.0379 & 0.0000 & 3.9781 & 20.0541 \\
\hline 5.2000 & 12.5801 & 3.1530 & 0.0346 & 0.0000 & 5.6504 & 18.2651 \\
\hline 5.4000 & 12.7283 & 3.3402 & 3.3287 & 0.0000 & 5.3211 & 21.3782 \\
\hline 6.2000 & 13.0475 & 3.3597 & 8.0038 & 0.0000 & 4.8584 & 25.9097 \\
\hline 8.0000 & 12.2323 & 2.3016 & 13.6204 & 0.0000 & 4.3058 & 30.1585 \\
\hline 8.2000 & 12.1233 & 2.2056 & 13.1853 & 0.0000 & 4.3084 & 29.6170 \\
\hline 9.4000 & 11.5017 & 1.7352 & 16.2071 & 0.0095 & 4.5816 & 32.2999 \\
\hline 9.6000 & 11.3777 & 1.6695 & 16.4122 & 0.0619 & 4.6119 & 32.4638 \\
\hline 10.0000 & 11.1255 & 1.5500 & 16.7163 & 0.2194 & 4.6096 & 32.6709 \\
\hline 12.0000 & 9.9804 & 1.1324 & 17.7046 & 1.1899 & 4.1247 & 32.9996 \\
\hline 14.0000 & 8.9622 & 0.8930 & 18.0313 & 1.9683 & 3.4726 & 32.4344 \\
\hline 18.0000 & 7.3501 & 0.6553 & 17.7249 & 2.8339 & 2.3905 & 30.2995 \\
\hline 22.0000 & 6.2279 & 0.5447 & 17.4088 & 3.1567 & 1.6629 & 28.4563 \\
\hline 26.0000 & 5.3772 & 0.4821 & 16.6850 & 3.2196 & 1.2002 & 26.4821 \\
\hline 30.0000 & 4.7285 & 0.4420 & 15.8581 & 3.1633 & 0.8980 & 24.6479 \\
\hline 35.0000 & 4.1232 & 0.4066 & 14.9840 & 3.0242 & 0.6539 & 22.7853 \\
\hline 40.0000 & 3.6695 & 0.3791 & 14.2927 & 2.8604 & 0.4965 & 21.3190 \\
\hline 50.0000 & 3.0272 & 0.3347 & 13.1214 & 2.5389 & 0.3177 & 19.0052 \\
\hline 60.0000 & 2.5890 & 0.2981 & 12.0010 & 2.2616 & 0.2239 & 17.0755 \\
\hline 80.0000 & 2.0283 & 0.2398 & 10.0366 & 1.8418 & 0.1292 & 14.0359 \\
\hline 100.0000 & 1.6842 & 0.1963 & 8.5716 & 1.5499 & 0.0813 & 11.8871 \\
\hline 110.0000 & 1.5566 & 0.1788 & 8.0767 & 1.4364 & 0.0706 & 11.1403 \\
\hline 120.0000 & 1.4294 & 0.1495 & 7.5859 & 1.4576 & 0.0686 & 10.5415 \\
\hline 140.0000 & 1.2578 & 0.1271 & 6.8041 & 1.2910 & 0.0444 & 9.3972 \\
\hline 170.0000 & 1.0703 & 0.1023 & 5.9139 & 1.1042 & 0.0207 & 8.1091 \\
\hline 190.0000 & 0.9753 & 0.0900 & 5.4391 & 1.0085 & 0.0097 & 7.4325 \\
\hline 200.0000 & 0.9344 & 0.0848 & 5.2269 & 0.9665 & 0.0051 & 7.1329 \\
\hline 275.0000 & 0.7151 & 0.0583 & 4.0722 & 0.7381 & 0.0000 & 5.5254 \\
\hline 425.0000 & 0.4937 & 0.0347 & 2.6654 & 0.5038 & 0.0000 & 3.6629 \\
\hline
\end{tabular}


TABLE 3. (Continued.)

\begin{tabular}{lcccccc}
\hline \hline Energy $(\mathrm{eV})$ & $\begin{array}{c}\text { Elastic ICS } \\
\left(\times 10^{-16} \mathrm{~cm}^{2}\right)\end{array}$ & $\begin{array}{c}\text { MTCS } \\
\left(\times 10^{-16} \mathrm{~cm}^{2}\right)\end{array}$ & $\begin{array}{c}\text { Excitation } \\
\left(\times 10^{-16} \mathrm{~cm}^{2}\right)\end{array}$ & $\begin{array}{c}\text { Direct ionization } \\
\text { ICS }\left(\times 10^{-16} \mathrm{~cm}^{2}\right)\end{array}$ & $\begin{array}{c}\text { Ps formation } \\
\left(\times 10^{-16} \mathrm{~cm}^{2}\right)\end{array}$ \\
\hline 650.0000 & 0.3417 & 0.0205 & 1.7082 & 0.3433 & 0.0000 \\
$\left(\times 10^{-16} \mathrm{~cm}^{2}\right)$
\end{tabular}

are in a reasonable qualitative accord although some differences in the magnitudes of the cross sections are noted. Of particular interest in Fig. 5(c) is a near-threshold structure that can be ascribed to excitation of the $2^{3} \mathrm{P}$ state of Be. In a nonrelativistic calculation, excitation of this state could only be via electron exchange, which is not available in positron scattering, and so one might a priori expect its cross section to be zero. However, in a relativistic framework (such as in our ROP results), this is not the case, due to configuration mixing, and although the interaction is quite weak, it will be nonzero as is shown in Fig. 5(c). Finally, we consider the TCS of Fig. 5(d). Most of the comments we made earlier in relation to the elastic ICS are equally valid here, except for the existence of a secondary maximum in a structure in the TCS at around $20 \mathrm{eV}$ (seen in both our OP and ROP results). This feature in Fig. 5(d) is not due to the

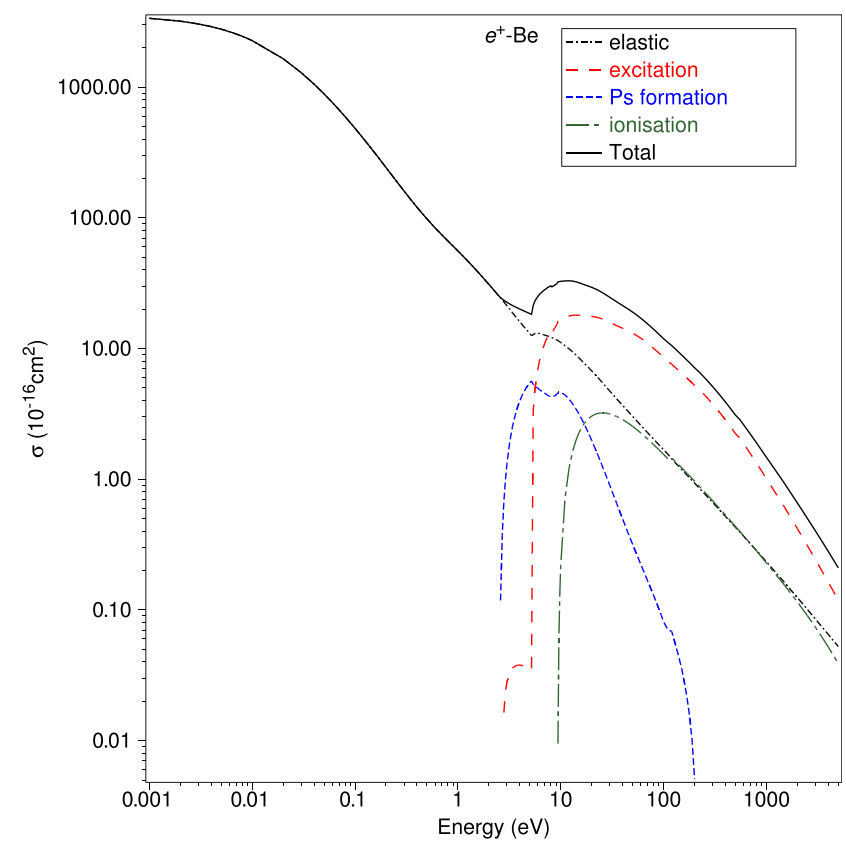

FIG. 3. The present ROP integral and total cross section results for positron scattering from beryllium. See the legend in the figure for the processes considered. existence of a resonance, rather if reflects the strong opening for the lowest-lying dipole-allowed $2{ }^{1} \mathrm{P}$ state in Be.

A similar comparison to that just given, but now for positron-Mg scattering, is made for the same scattering processes in Fig. 6. In this case, however, at the TCS level [Fig. 6(e)], we at least have experimental results from the Wayne State University group ${ }^{7-9}$ which are embodied in the recommended data from Ratnavelu et al. ${ }^{2}$ That data predicts a strong low-energy resonance feature, which is well reproduced by the theoretical results from the computations of Poveda et al., ${ }^{6}$ Mitroy et al., ${ }^{10}$ and the $\mathrm{CCC}^{12}$ but not by our ROP or the results of Bromley et al. ${ }^{3}$ Indeed, to within the $\pm 20 \%$ data uncertainty cited by Ratnavelu et al., ${ }^{2}$ the level of accord between their recommended data and the CCC, over the common energy regime, is excellent [see Fig. 6(e)]. Note that for incident positron energies greater than about $20 \mathrm{eV}$, we highlight the very good level of agreement between our OP and ROP calculations and the CCC. This augurs very well for constructing a recommended higher energy TCS for positron-Mg scattering (see Sec. 4). Much of what we have just noted for the TCS is also valid for the elastic ICS [Fig. 6(a)], and so we do not discuss it further here. Considering Fig. 6(b), for the positronium formation ICS, we find theoretical results from Gribakin et al.,${ }^{13}$ Hewitt et al.,${ }^{14}$ Chang and Zhou, ${ }^{15}$ the CCC, ${ }^{12}$ and our OP and ROP calculations. It is clear from this figure [Fig. 6(b)] that there is quite a spread in the ICS magnitudes for this process, although with the exception of the Hewitt et al. ${ }^{14}$ result, which is quasi-isotropic, the qualitative energy dependence of all the theories is quite similar. One interesting observation from Fig. 6(d) is that both our OP and ROP calculations predict the positronium formation cross section to persist to somewhat higher energies than that found in the 2 -center CCC result. ${ }^{12}$ In the case of He, the eigen 2-electron system (both $\mathrm{Be}$ and $\mathrm{Mg}$ can be considered as quasi-2electron systems ${ }^{22,23}$ ), Ratnavelu et al. ${ }^{2}$ recommended a positronium formation cross section, based on measurements, that persists out to about $200 \mathrm{eV}$. This energy range in $\mathrm{He}$ is more consistent, in this case, with the present $\mathrm{OP}$ and ROP computations than that from the CCC for Mg: In Fig. 6(c), we only have the OP and ROP results available for the ICSs for the sum over all discrete electronic-state excitations. Here, we note that our OP method has a problem ${ }^{23}$ in that there is still an uncertainty as to which excitation threshold $\left(3^{3} \mathrm{P}\right.$ or $3^{1} \mathrm{P}$ state) energy is the appropriate one to employ (see Sec. 2 ). This arises as while the $3^{3} \mathrm{P}$ transition is supposed to be forbidden, it is in fact optically observed in $\mathrm{Mg}$. As a consequence, at this time 
TABLE 4. A selection of the present ROP results $\left(\times 10^{-16} \mathrm{~cm}^{2}\right)$ for positron scattering from magnesium

\begin{tabular}{|c|c|c|c|c|c|c|}
\hline Energy $(\mathrm{eV})$ & $\begin{array}{c}\text { Elastic ICS } \\
\left(\times 10^{-16} \mathrm{~cm}^{2}\right)\end{array}$ & $\begin{array}{c}\text { MTCS } \\
\left(\times 10^{-16} \mathrm{~cm}^{2}\right)\end{array}$ & $\begin{array}{c}\text { Excitation } \\
\left(\times 10^{-16} \mathrm{~cm}^{2}\right)\end{array}$ & $\begin{array}{l}\text { Direct ionization ICS } \\
\quad\left(\times 10^{-16} \mathrm{~cm}^{2}\right)\end{array}$ & $\begin{array}{l}\text { Ps formation } \\
\left(\times 10^{-16} \mathrm{~cm}^{2}\right)\end{array}$ & $\begin{array}{c}\text { Total } \\
\left(\times 10^{-16} \mathrm{~cm}^{2}\right)\end{array}$ \\
\hline 0.0000 & 695.5280 & 695.5280 & 0.0000 & 0.0000 & 0.0000 & 695.5280 \\
\hline 0.0010 & 732.1410 & 745.4850 & 0.0000 & 0.0000 & 0.0000 & 732.1410 \\
\hline 0.0800 & 463.6440 & 510.4020 & 0.0000 & 0.0000 & 0.0000 & 463.6440 \\
\hline 0.1200 & 389.8210 & 408.8210 & 0.0000 & 0.0000 & 0.0000 & 389.8210 \\
\hline 0.2000 & 311.3940 & 270.7770 & 0.0000 & 0.0000 & 0.0000 & 311.3940 \\
\hline 0.2770 & 273.5100 & 189.3430 & 0.0000 & 0.0000 & 0.0000 & 273.5100 \\
\hline 0.3400 & 252.2920 & 146.1530 & 0.0000 & 0.0000 & 0.0000 & 252.2920 \\
\hline 0.4200 & 229.8870 & 110.4930 & 0.0000 & 0.0000 & 0.0000 & 229.8870 \\
\hline 0.5000 & 209.8140 & 88.0610 & 0.0000 & 0.0000 & 0.0000 & 209.8140 \\
\hline 0.6500 & 176.9180 & 64.2350 & 0.0000 & 0.0000 & 0.0000 & 176.9180 \\
\hline 0.8750 & 138.7268 & 46.3253 & 0.0000 & 0.0000 & 0.4688 & 139.1956 \\
\hline 0.9000 & 134.8134 & 44.7053 & 0.0000 & 0.0000 & 1.0150 & 135.8284 \\
\hline 1.2000 & 97.2886 & 29.8558 & 0.0000 & 0.0000 & 8.7145 & 106.0031 \\
\hline 1.4000 & 81.2173 & 23.7729 & 0.0000 & 0.0000 & 12.5755 & 93.7928 \\
\hline 1.6000 & 69.7110 & 19.5057 & 0.0000 & 0.0000 & 15.4744 & 85.1854 \\
\hline 2.0000 & 54.7187 & 14.1248 & 0.0000 & 0.0000 & 19.2659 & 73.9846 \\
\hline 2.4000 & 45.5362 & 10.9924 & 0.0000 & 0.0000 & 21.3680 & 66.9042 \\
\hline 2.8000 & 39.3450 & 8.9747 & 0.0678 & 0.0000 & 22.4843 & 61.8971 \\
\hline 2.9000 & 38.1001 & 8.5771 & 0.0955 & 0.0000 & 22.6591 & 60.8547 \\
\hline 3.2000 & 34.8979 & 7.5600 & 0.1420 & 0.0000 & 23.0410 & 58.0809 \\
\hline 3.8000 & 30.1580 & 6.0746 & 0.1796 & 0.0000 & 23.3413 & 53.6789 \\
\hline 4.2000 & 27.8007 & 5.3484 & 0.1902 & 0.0000 & 23.3273 & 51.3182 \\
\hline 4.4000 & 27.6535 & 5.3987 & 2.5881 & 0.0000 & 21.6394 & 51.8810 \\
\hline 4.6000 & 27.7982 & 5.5302 & 4.6974 & 0.0000 & 20.2001 & 52.6957 \\
\hline 5.2000 & 26.6429 & 4.9959 & 8.8294 & 0.0000 & 17.8467 & 53.3190 \\
\hline 6.4000 & 24.3225 & 3.7677 & 14.2539 & 0.0000 & 15.2958 & 53.8723 \\
\hline 7.8000 & 22.0605 & 2.7576 & 17.9762 & 0.0505 & 14.6376 & 54.7249 \\
\hline 8.0000 & 21.7609 & 2.6453 & 18.3502 & 0.1683 & 14.5144 & 54.7937 \\
\hline 8.2000 & 21.4631 & 2.5397 & 18.6669 & 0.3160 & 14.3710 & 54.8171 \\
\hline 10.0000 & 18.9303 & 1.8302 & 19.8088 & 1.9252 & 12.8590 & 53.5232 \\
\hline 12.0000 & 16.6769 & 1.3834 & 20.2225 & 3.3747 & 11.1402 & 51.4143 \\
\hline 14.0000 & 14.8845 & 1.1173 & 20.4804 & 4.3527 & 9.6210 & 49.3386 \\
\hline 18.0000 & 12.1604 & 0.8360 & 19.7662 & 5.3781 & 7.4452 & 44.7499 \\
\hline 22.0000 & 10.3012 & 0.7044 & 18.8894 & 5.7417 & 5.9063 & 40.8387 \\
\hline 30.0000 & 7.9671 & 0.5867 & 17.6104 & 5.7117 & 3.9757 & 35.2649 \\
\hline 40.0000 & 6.2703 & 0.5206 & 15.8525 & 5.2545 & 2.7640 & 30.1414 \\
\hline 45.0000 & 5.6913 & 0.4982 & 14.9474 & 5.0007 & 2.3342 & 27.9737 \\
\hline 55.0000 & 4.8369 & 0.4573 & 13.3604 & 4.5213 & 1.7346 & 24.4532 \\
\hline 65.0000 & 4.2345 & 0.4184 & 12.0858 & 4.1166 & 1.3486 & 21.7855 \\
\hline 85.0000 & 3.4404 & 0.3537 & 10.2050 & 3.5090 & 0.8640 & 18.0184 \\
\hline 100.0000 & 3.0376 & 0.3100 & 9.1515 & 3.2093 & 0.6370 & 16.0354 \\
\hline 120.0000 & 2.6544 & 0.2706 & 8.2710 & 2.8767 & 0.4197 & 14.2218 \\
\hline 140.0000 & 2.3744 & 0.2409 & 7.4477 & 2.6107 & 0.2636 & 12.6963 \\
\hline 160.0000 & 2.1600 & 0.2178 & 6.7563 & 2.3928 & 0.1553 & 11.4644 \\
\hline 180.0000 & 1.9896 & 0.1992 & 6.1625 & 2.2108 & 0.0787 & 10.4417 \\
\hline 190.0000 & 1.9168 & 0.1912 & 5.8955 & 2.1304 & 0.0489 & 9.9917 \\
\hline 200.0000 & 1.8507 & 0.1840 & 5.6460 & 2.0560 & 0.0235 & 9.5762 \\
\hline 300.0000 & 1.4119 & 0.1345 & 3.9553 & 1.5338 & 0.0000 & 6.9010 \\
\hline 400.0000 & 1.1722 & 0.1070 & 2.9165 & 1.2252 & 0.0000 & 5.3138 \\
\hline 500.0000 & 1.0167 & 0.0888 & 2.2601 & 1.0165 & 0.0000 & 4.2933 \\
\hline 650.0000 & 0.8605 & 0.0705 & 1.7154 & 0.8266 & 0.0000 & 3.4025 \\
\hline
\end{tabular}


TABLE 4. (Continued.)

\begin{tabular}{lccccc}
\hline \hline Energy $(\mathrm{eV})$ & $\begin{array}{c}\text { Elastic ICS } \\
\left(\times 10^{-16} \mathrm{~cm}^{2}\right)\end{array}$ & $\begin{array}{c}\text { MTCS } \\
\left(\times 10^{-16} \mathrm{~cm}^{2}\right)\end{array}$ & $\begin{array}{c}\text { Excitation } \\
\left(\times 10^{-16} \mathrm{~cm}^{2}\right)\end{array}$ & $\begin{array}{c}\text { Direct ionization ICS } \\
\left(\times 10^{-16} \mathrm{~cm}^{2}\right)\end{array}$ & $\begin{array}{c}\text { Ps formation } \\
\left(\times 10^{-16} \mathrm{~cm}^{2}\right)\end{array}$ \\
\hline 850.0000 & 0.7244 & 0.0546 & 1.2318 & 0.6470 & 0.0000 \\
$\left(\times 10^{-16} \mathrm{~cm}^{2}\right)$
\end{tabular}

only our ROP result can be considered further in Sec. 4 . The results in Fig. 6(d), for the ionization ICS, are, however, very encouraging. Here, our OP calculation and the CCC result ${ }^{12}$ are in very good agreement over their common energy range. Similarly, while our ROP ionization ICS has a slightly lower magnitude than the OP and CCC, in the energy region about the peak of this cross section, its overall accord with the CCC and OP results is actually quite good. As a consequence, in Sec. 4, we anticipate being able to construct a robust recommended ICS dataset for this process.

\section{Recommended Data}

Based largely on the discussion in Sec. 3, here, we now construct our recommended cross section datasets for positron-Be and positron-Mg scattering in the gas phase.

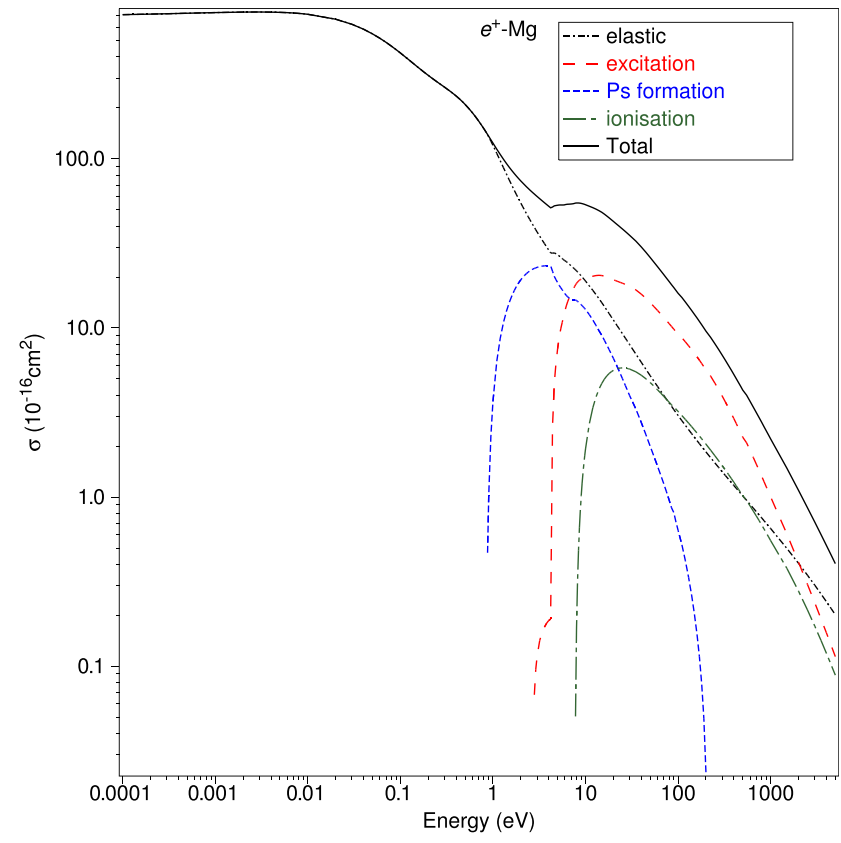

FIG. 4. The present ROP integral and total cross section results for positron scattering from magnesium. See the legend in the figure for the processes considered.
For positron-Be scattering, we form the recommended elastic ICS by taking an average of the Mitroy and Ivanov, ${ }^{4}$ Poveda et al., ${ }^{6}$ and Reid and Wadehra ${ }^{5}$ results up to $0.3 \mathrm{eV}$ and then mapping that onto our OP calculation (scaling factor $=0.946$ ) to extend the results up to $5000 \mathrm{eV}$. This is effectively the method of Itikawa, who has established a reputation for the critical analysis and forming recommended cross section data in electron-molecule scattering (see, e.g., Refs. 37 and 38). Given the level of accord between the various calculations (see Fig. 5), over their overlapping energy ranges, a conservative estimate of $\pm 20 \%$ in the uncertainty of the elastic ICS seems reasonable. In the case of positronium formation, we only have the present ROP and OP results available to form a recommended dataset. Here, our OP calculation ICS does not appear to be correctly trending to the positronium formation energy threshold, and as a result, we simply employ our ROP result as our recommended data. In their recent paper, McEachran and Stauffer ${ }^{39}$ found that for positron-helium scattering, their ROP result was, at worst, to within $\pm 25 \%$ of the most recent measured cross section from the Australian National University (ANU) group. ${ }^{40}$ As a consequence, we adopt an uncertainty of $\pm 25 \%$ on our positronium formation ICS in Be. In the case of the sum over all the discrete-excitation electronic-states, we again prefer our ROP result, due to the uncertainty in the correct electronic-state threshold to be applied in our OP computations (see Sec. 2). We do not have a strong guidance here in estimating the uncertainty on those ICSs, and so we opt for a conservative estimate of $\pm 40 \%$. For the recommended ionization ICS, we again apply the Itikawa approach, by taking an average of our OP and ROP results. From $10 \mathrm{eV}$ until threshold, however, to ensure continuity with that average, we use our ROP calculation scaled by a factor of 0.83 . In this case, the uncertainty is taken to be the difference between the ROP and OP results, on the one hand, and our recommended ionization ICS on the other hand, which is typically $\pm 25 \%$. Finally, the recommended TCS for positron-Be scattering is simply obtained, at each given incident positron energy, by adding up the elastic ICS, the positronium formation ICS, the ICS for the sum of the discrete-excitation electronic-states, and the ionization ICS. Our estimated uncertainty on these recommended TCS is $\pm 25 \%$. All the Be results from the above process are plotted in Fig. 7(a) and listed in Table 5.

Let us now consider Mg. Remembering that below the positronium formation threshold energy the total cross section is equivalent to the elastic ICS, the recommended TCS of Ratnavelu et al. ${ }^{2}$ also 

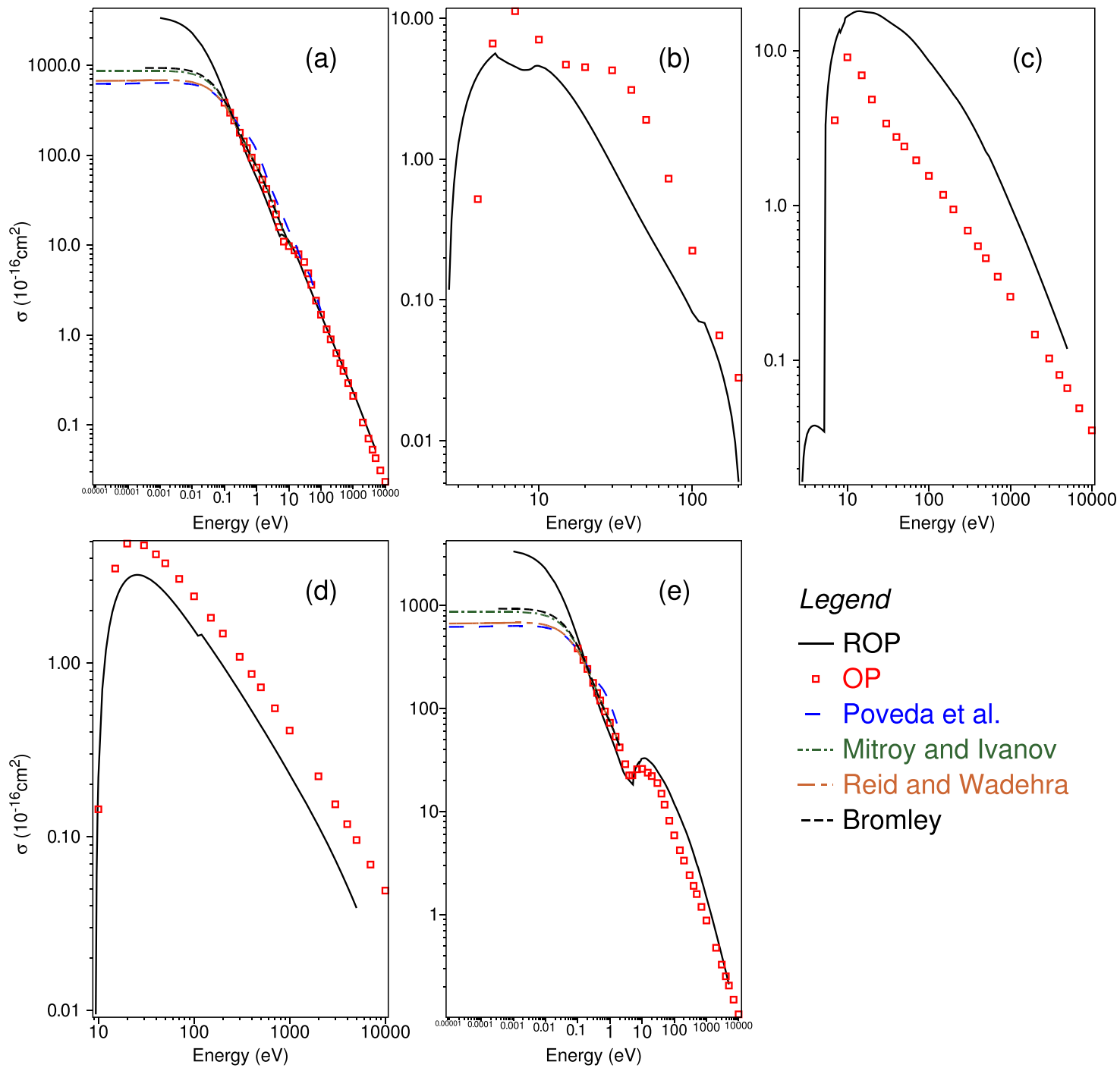

Legend

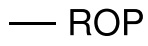

- OP

- Poveda et al.

-.-- Mitroy and Ivanov

- - Reid and Wadehra

--- Bromley

FIG. 5. Comparison of the present OP and ROP integral and total cross section results, against those from earlier studies, for positron-Be scattering. (a) Elastic scattering, (b) positronium formation, (c) sum over discrete electronic-state excitation, (d) ionization, and (e) total scattering. See the legend in the figure and text.

provides a guide to its elastic ICS below $E_{\mathrm{Ps}}$. That comparison suggests that the CCC elastic ICS is quantitatively reproducing this cross section at lower energies. Hence, we base our recommended elastic ICS around the CCC, with an extrapolation to very low energies using the form of the results from Poveda et al. ${ }^{6}$ (suitably scaled, $\times 1.33$, to map smoothly onto the CCC result) and on extrapolation from $50 \mathrm{eV}$ to $5000 \mathrm{eV}$ by employing our ROP result scaled by a factor of 0.88 . We estimate the uncertainty in this data to be $\pm 20 \%$, consistent with Ratnavelu et $a l^{2}$ for the TCS. Note that the CCC approach for positron-atom scattering was previously shown to provide a good description of the low-energy scattering dynamics by the Trento group, ${ }^{41-43}$ with the behavior in Mg being entirely consistent with that observation. For the case of positronium formation, there are several theoretical results available in the literature. ${ }^{12-15}$ Of these, the results from the 2-center CCC computation ${ }^{12}$ and Gribakin et al. ${ }^{13}$ are the most comprehensive. We therefore form our recommended $\mathrm{Mg}$ positronium formation ICS from an average of the CCC, Gribakin et al., ${ }^{13}$ and our ROP results over the energy range where they overlap. At lower energies, we extrapolate to the positronium formation threshold using the ROP computation with a suitable scaling $(\times 1.32)$ for continuity, while for higher energies (up to $50 \mathrm{eV}$ where the CCC stops), a suitably scaled $(\times 1.13)$ average of the CCC and ROP results is employed. Beyond $50 \mathrm{eV}$, the form of the ROP (scaled by $\times 0.66$ for continuity) is used. Given the spread in the available theoretical results [see Fig. 6(b)], for this process, a conservative uncertainty of $\pm 30 \%$ is assigned to our recommended positronium formation ICSs. Only the present OP and ROP results are available for the sum over all discrete-excitation electronic state ICSs, and given our earlier 

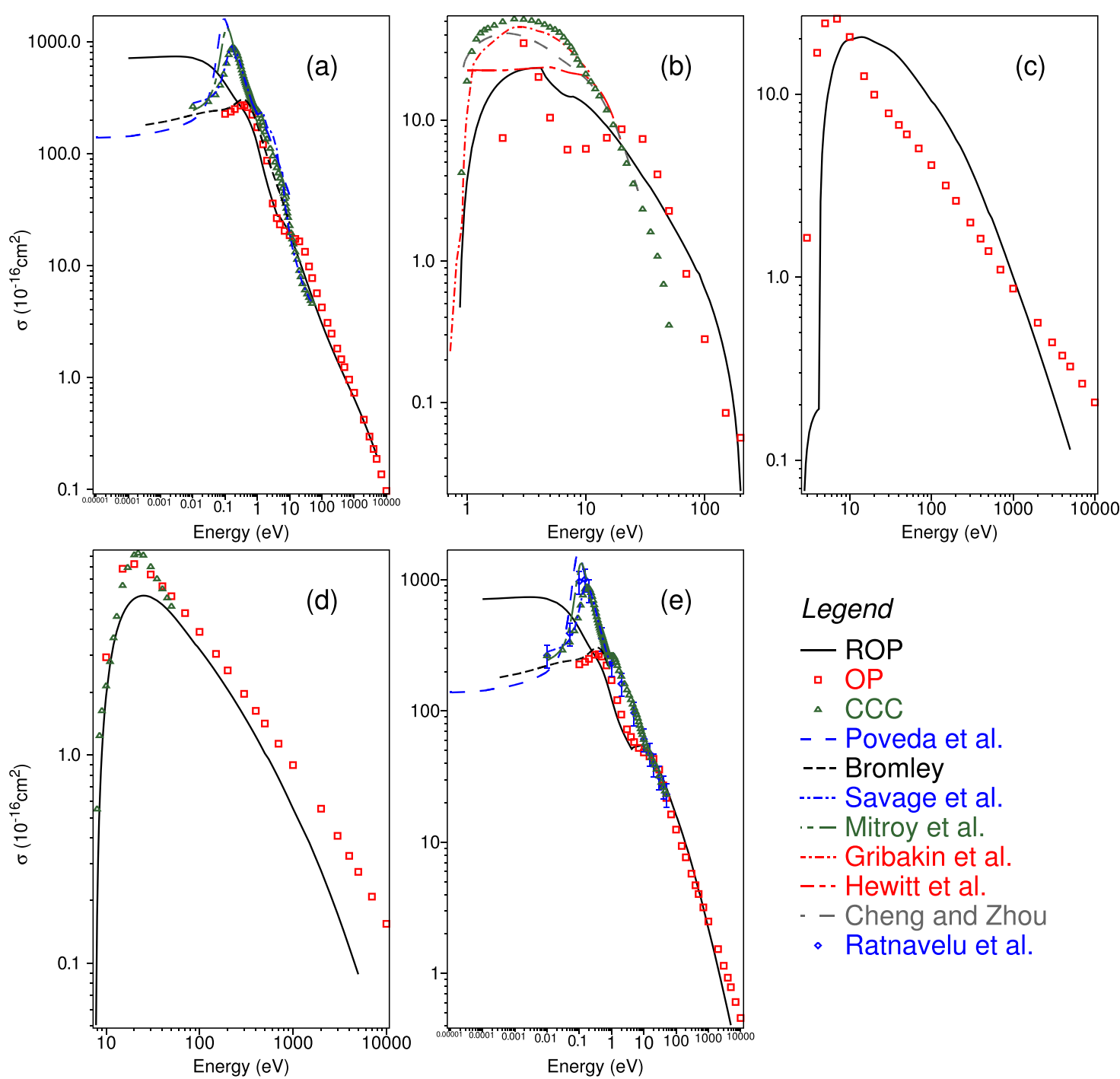

Legend

- ROP

- OP

$\triangle \mathrm{CCC}$

- - Poveda et al.

--- Bromley

.... Savage et al.

- - Mitroy et al.

-... Gribakin et al.

-. Hewitt et al.

- - Cheng and Zhou

- Ratnavelu et al.

FIG. 6. Comparison of the present OP and ROP integral and total cross section results, against those from earlier studies, for positron-Mg scattering. (a) Elastic scattering, (b) positronium formation, (c) sum over discrete electronic-state excitation, (d) ionization, and (e) total scattering. See also legend in the figure and text.

discussion in Sec. 3 for this process, here we simply adopt the ROP result to be our recommended ICS in Mg. Similar to the case for positron-Be scattering just discussed above, an uncertainty of $\pm 40 \%$ is given on these cross sections. Following once again the method of Itikawa, ${ }^{37,38}$ for the ionization ICS we take an average of the CCC, OP, and $\mathrm{ROP}$ results, up to $50 \mathrm{eV}$, to form our recommended cross section. Above $50 \mathrm{eV}$, where the CCC stops, and up to $5000 \mathrm{eV}$, we employ an average of our OP and ROP results, suitably scaled by a factor of 0.99 to ensure continuity at $50 \mathrm{eV}$. Note that the ROP result, scaled by 3.37 , was employed to extend the ICS down to threshold from $10 \mathrm{eV}$. In this case, the rather good overall agreement between the three theoretical results enables us to assign a $\pm 20 \%$ uncertainty to our recommended ionization ICS. The recommended TCS for positron-Mg scattering is then subsequently determined by simply adding up all the preceding recommended ICSs, with the uncertainty estimate on these data being $\pm 20 \%$ (consistent with that from Ratnavelu et al. ${ }^{2}$ ). All the recommended data from this discussion can be found in Table 6, and they are also all plotted in Fig. 7(b).

\section{Transport Simulations}

While we had previously simulated electron transport behavior for a swarm of electrons in $\mathrm{Mg}{ }^{23}$ we had not done so in Be. Therefore, we start this section by looking at the comparative electron transport in Be and $\mathrm{Mg}$, before repeating that discussion but now for positron transport in Be and $\mathrm{Mg}$. We reiterate that these new positron simulations have been undertaken with the recommended positron-Be and positron-Mg cross section database we determined in Sec. 4. We 

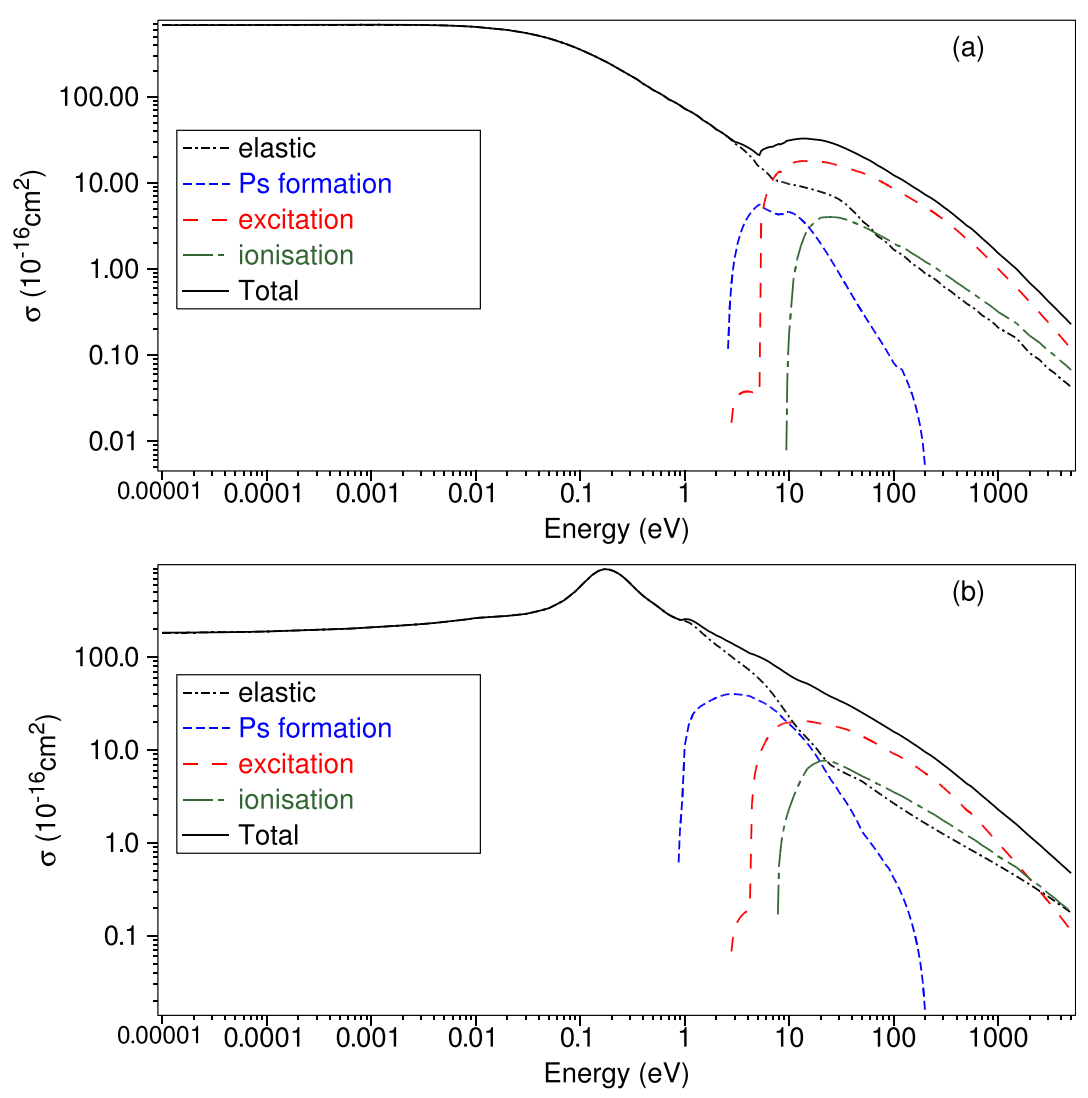

FIG. 7. Recommended integral and total cross sections for positron scattering from (a) Be and (b) Mg. See legend in the figure and text for further details.

highlight that in all calculations for both metal vapors, we have assumed isotropic scattering in the electronic excitation and ionization processes, while we have included the anisotropic nature of elastic scattering through the use of the momentum transfer cross section. The positron elastic MTCS for Be and Mg is implemented directly as per Tables 3 and 4, while for electrons in Be, we have assumed the form of the ROP differential cross section to scale the recommended integral elastic cross section in order to obtain a recommended elastic MTCS. Finally, for each species, a comparison of the positron versus electron transport behavior is presented and discussed. In what follows, we implement a well benchmarked multiterm solution of Boltzmann's equation ${ }^{44}$ for the calculation of the transport coefficients in gaseous $\mathrm{Be}$ and $\mathrm{Mg}$, and we have found that the two-term approximation ${ }^{45,46}$ is generally sufficient for the coefficients presented, over the range of $E / n_{0}$ considered. Our physical discussions below will focus on the rates and the drift velocities.

\subsection{Electron transport in Be}

In Fig. 8 , we present the results for the reduced ionization rate $k_{\text {io }} / n_{0}$, where $n_{0}$ is the neutral density, and the bulk and flux drift velocities for an electron swarm in Be vapor over the range of reduced fields, $E / n_{0}$, from 0.001 to $1000 \mathrm{Td}\left(1 \mathrm{Td}=10^{21} \mathrm{~V} \mathrm{~m}^{2}\right)$. The results are compared with those for electron swarms in $\mathrm{Mg}$ vapor ${ }^{23}$ at the same temperature of $750 \mathrm{~K}$.

In the low field regime (up to $4 \mathrm{Td}$ ), we observe that the flux drift velocity of electrons in Be is substantially higher than that in $\mathrm{Mg}$. This reflects the reduced elastic momentum transfer cross section of Be in the thermal-energy regime $(\sim 0.1 \mathrm{eV})$. This relation is reversed at higher $E / n_{0}$ as the elastic momentum transfer rate for electrons in Be substantially increases relative to $\mathrm{Mg}$ at the higher energies. This is also reflected in the mean energy (not shown) where the profile for Be lies above that for $\mathrm{Mg}$ for the majority of the $E / n_{0}$ range considered. At low $E / n_{0}$, this is in response to both the reduced elastic and electronic excitation collisional rates for electrons in $\mathrm{Be}$ as compared to $\mathrm{Mg}$, while for higher $E / n_{0}$, this is impacted upon by the ionization processes. Below $50 \mathrm{Td}$, the electronic excitation rates for electrons in Be are significantly lower than those in $\mathrm{Mg}$; however, above $50 \mathrm{Td}$, there is very little difference in these rates. While the threshold for ionization is lower in $\mathrm{Be}$ as compared to $\mathrm{Mg}$, the enhanced power input into Be results in the initiation of ionization processes at slightly lower $E / n_{0}$ than $\mathrm{Mg}$. The presence of significant ionization manifests itself in differences between the bulk and flux drift velocities at higher $E / n_{0}$ (e.g., above a few hundred Td). The ionization process results in the generation of electrons predominantly at the front of the swarm, resulting in a shifting of the center of mass of the swarm in the 
TABLE 5. Recommended cross sections for positron scattering from Be. The errors on the elastic ICS are $\pm 20 \% ; \pm 25 \%$ on the positronium formation ICS; an uncertainty of $\pm 40 \%$ on the sum of the discrete-excitation electronic-states (inelastic); $\pm 25 \%$ on the ionization ICS, and $\pm 25 \%$ on the TCS

\begin{tabular}{|c|c|c|c|c|c|}
\hline Energy (eV) & $\begin{array}{c}\text { Elastic ICS } \\
\left(\times 10^{-16} \mathrm{~cm}^{2}\right)\end{array}$ & $\begin{array}{l}\text { Ps formation } \\
\left(\times 10^{-16} \mathrm{~cm}^{2}\right)\end{array}$ & $\begin{array}{c}\text { Excitation } \\
\left(\times 10^{-16} \mathrm{~cm}^{2}\right)\end{array}$ & $\begin{array}{l}\text { Direct ionization ICS } \\
\quad\left(\times 10^{-16} \mathrm{~cm}^{2}\right)\end{array}$ & $\begin{array}{c}\text { Total } \\
\left(\times 10^{-16} \mathrm{~cm}^{2}\right)\end{array}$ \\
\hline $1.000000 \times 10^{-5}$ & 680.985 & 0.000000 & 0.000000 & 0.000000 & 680.985 \\
\hline $8.900000 \times 10^{-5}$ & 683.819 & 0.000000 & 0.000000 & 0.000000 & 683.819 \\
\hline $1.000000 \times 10^{-3}$ & 688.764 & 0.000000 & 0.000000 & 0.000000 & 688.764 \\
\hline $2.000000 \times 10^{-2}$ & 598.917 & 0.000000 & 0.000000 & 0.000000 & 598.917 \\
\hline $8.000000 \times 10^{-2}$ & 395.328 & 0.000000 & 0.000000 & 0.000000 & 395.328 \\
\hline 0.160000 & 271.286 & 0.000000 & 0.000000 & 0.000000 & 271.286 \\
\hline 0.250000 & 201.686 & 0.000000 & 0.000000 & 0.000000 & 201.686 \\
\hline 0.400000 & 141.960 & 0.000000 & 0.000000 & 0.000000 & 141.960 \\
\hline 0.700000 & 93.8000 & 0.000000 & 0.000000 & 0.000000 & 93.8000 \\
\hline 1.20000 & 65.0720 & 0.000000 & 0.000000 & 0.000000 & 65.0720 \\
\hline 2.60000 & 34.1040 & 0.118300 & 0.000000 & 0.000000 & 34.2223 \\
\hline 2.70000 & 32.7880 & 0.371000 & 0.000000 & 0.000000 & 33.1590 \\
\hline 2.80000 & 31.4720 & 0.670700 & $1.640000 \times 10^{-2}$ & 0.000000 & 32.1591 \\
\hline 2.90000 & 30.1560 & 0.989800 & $2.370000 \times 10^{-2}$ & 0.000000 & 31.1695 \\
\hline 3.00000 & 28.8400 & 1.31400 & $2.810000 \times 10^{-2}$ & 0.000000 & 30.1821 \\
\hline 3.40000 & 26.0848 & 2.53810 & $3.590000 \times 10^{-2}$ & 0.000000 & 28.6588 \\
\hline 4.20000 & 20.7424 & 4.35360 & $3.760000 \times 10^{-2}$ & 0.000000 & 25.1336 \\
\hline 5.20000 & 15.4056 & 5.65040 & $3.460000 \times 10^{-2}$ & 0.000000 & 21.0906 \\
\hline 5.40000 & 14.9072 & 5.32110 & 3.32870 & 0.000000 & 23.5570 \\
\hline 5.60000 & 14.4088 & 5.18780 & 4.77270 & 0.000000 & 24.3693 \\
\hline 6.20000 & 12.9136 & 4.85840 & 8.00380 & 0.000000 & 25.7758 \\
\hline 7.00000 & 10.9200 & 4.54940 & 10.9910 & 0.000000 & 26.4604 \\
\hline 8.00000 & 10.5187 & 4.30580 & 13.6204 & 0.000000 & 28.4449 \\
\hline 8.20000 & 10.4384 & 4.30840 & 13.1853 & 0.000000 & 27.9321 \\
\hline 9.40000 & 9.95680 & 4.58160 & 16.2071 & $7.865861 \times 10^{-3}$ & 30.7534 \\
\hline 9.60000 & 9.87653 & 4.61190 & 16.4122 & $5.125230 \times 10^{-2}$ & 30.9519 \\
\hline 9.80000 & 9.79627 & 4.61880 & 16.5771 & 0.111612 & 31.1038 \\
\hline 10.0000 & 9.71600 & 4.60960 & 16.7163 & 0.181660 & 31.2236 \\
\hline 13.0000 & 9.11120 & 3.79480 & 17.9754 & 1.88533 & 32.7667 \\
\hline 15.0000 & 8.70800 & 3.16900 & 17.9675 & 2.87980 & 32.7243 \\
\hline 20.0000 & 7.89600 & 1.98570 & 17.6137 & 3.95645 & 31.4518 \\
\hline 26.0000 & 7.03920 & 1.20020 & 16.6850 & 4.01220 & 28.9366 \\
\hline 30.0000 & 6.46800 & 0.898000 & 15.8581 & 3.96165 & 27.1858 \\
\hline 35.0000 & 5.65600 & 0.653900 & 14.9840 & 3.75910 & 25.0530 \\
\hline 40.0000 & 4.84400 & 0.496500 & 14.2927 & 3.54420 & 23.1774 \\
\hline 45.0000 & 4.22800 & 0.391100 & 13.6935 & 3.34275 & 21.6553 \\
\hline 50.0000 & 3.61200 & 0.317700 & 13.1214 & 3.14545 & 20.1966 \\
\hline 60.0000 & 3.01000 & 0.223900 & 12.0010 & 2.83180 & 18.0667 \\
\hline 70.0000 & 2.40800 & 0.167100 & 10.9566 & 2.54190 & 16.0736 \\
\hline 80.0000 & 2.16533 & 0.129200 & 10.0366 & 2.34190 & 14.6730 \\
\hline 100.000 & 1.68000 & $8.130000 \times 10^{-2}$ & 8.57160 & 1.98595 & 12.3188 \\
\hline 110.000 & 1.57528 & $7.060000 \times 10^{-2}$ & 8.07670 & 1.86928 & 11.5919 \\
\hline 120.000 & 1.47056 & $6.860000 \times 10^{-2}$ & 7.58590 & 1.81996 & 10.9450 \\
\hline 130.000 & 1.36584 & $5.500000 \times 10^{-2}$ & 7.17030 & 1.71594 & 10.3071 \\
\hline 150.000 & 1.15640 & $3.550000 \times 10^{-2}$ & 6.47730 & 1.52235 & 9.19155 \\
\hline 170.000 & 1.05112 & $2.070000 \times 10^{-2}$ & 5.91390 & 1.39462 & 8.38034 \\
\hline 190.000 & 0.945840 & $9.700000 \times 10^{-3}$ & 5.43910 & 1.27789 & 7.67253 \\
\hline 200.000 & 0.893200 & $5.100000 \times 10^{-3}$ & 5.22690 & 1.22245 & 7.34765 \\
\hline 300.000 & 0.627200 & 0.000000 & 3.76600 & 0.885450 & 5.27865 \\
\hline 400.000 & 0.484400 & 0.000000 & 2.84090 & 0.698550 & 4.02385 \\
\hline 500.000 & 0.397600 & 0.000000 & 2.23150 & 0.580300 & 3.20940 \\
\hline
\end{tabular}


TABLE 5. (Continued.)

\begin{tabular}{lllcc}
\hline \hline Energy $(\mathrm{eV})$ & $\begin{array}{c}\text { Elastic ICS } \\
\left(\times 10^{-16} \mathrm{~cm}^{2}\right)\end{array}$ & $\begin{array}{c}\text { Ps formation } \\
\left(\times 10^{-16} \mathrm{~cm}^{2}\right)\end{array}$ & $\begin{array}{c}\text { Excitation } \\
\left(\times 10^{-16} \mathrm{~cm}^{2}\right)\end{array}$ & $\begin{array}{c}\text { Direct ionization ICS } \\
\left(\times 10^{-16} \mathrm{~cm}^{2}\right)\end{array}$ \\
\hline 700.000 & 0.291200 & 0.000000 & 1.56470 & 0.434650 \\
$\left(\times 10^{-16} \mathrm{~cm}^{2}\right)$
\end{tabular}

direction of the drift velocity and a subsequent enhancement of the bulk drift velocity over the flux drift velocity. Given the near equivalence of the ionization rates for both gases, the modifications to the bulk drift velocity in both gases are also essentially equivalent.

\subsection{Positron transport in gaseous $\mathrm{Be}$ and $\mathrm{Mg}$}

In Fig. 9, we present and compare the results for positron transport in gaseous $\mathrm{Mg}$ and $\mathrm{Be}$. There are fundamental differences in the nature of the collisional processes available to positrons (as compared with electrons). First, positronium (Ps) formation is a direct loss-channel for positron swarms. Second, positron-induced ionization is a particle conserving process in contrast to the electron case. While Ps formation can be treated like an attachment process from a transport theory viewpoint, positron-impact ionization requires a different collision operator. ${ }^{47}$ There is generally sensitivity in the transport coefficients as to how the excess energy is shared between the scattered positron and ejected electron in an ionization process; however, in the absence of data on this, in what follows, we assume that all fractions of energy sharing are equally likely.

In Fig. 9, for positron transport in Be, we observe that Psformation becomes operative at a few $\mathrm{Td}$. In contrast, direct positron impact ionization is observed at a few hundred Td reflecting the substantial difference in the threshold energies for these processes. For the range of $E / n_{0}$ considered, the positronium formation rate exceeds the positron-impact direct ionization rate, reflecting the dominance of the Ps-formation cross section over the positronimpact direct ionization cross section in the energy regions up to approximately $15 \mathrm{eV}$. For sufficiently high $E / n_{0}$, we would expect this relation to invert. For positrons in $\mathrm{Mg}$, we observe that Ps-formation is operative at all $E / n_{0}$ sampled, indicative of its presence at lower energies and its increased magnitude (which is of an order of magnitude higher) than for Be. Positron impact ionization in $\mathrm{Mg}$ is operative at higher $E / n_{0}$ than for Be reflecting the slightly lower threshold energy for this process, but more importantly the reduced mean energy for positrons in $\mathrm{Mg}$ as compared with Be due to the enhanced elastic and inelastic scattering rates in $\mathrm{Mg}$.

The flux drift velocity for positrons in Be initially increases linearly with $E / n_{0}$ before a region of rapid increase in the flux drift velocity (and mean energy - not shown) around 2-6 Td, reflecting the significant decrease in elastic cross section magnitude with energy in this region. The Ps-formation channel then opens which, in turn, modifies the center-of-mass motion of the swarm through selective loss of positrons from the high-energy front of the swarm. This manifests itself in a bulk drift velocity that is less than the flux drift velocity. Indeed, in this field region, the flux drift velocity can be in excess of an order of magnitude greater than the bulk drift velocity. More importantly, a slight negative differential conductivity (NDC-the reduction of drift velocity with increasing $E / n_{0}$ ) emerges over an extended $E / n_{0}$ range from $2 \mathrm{Td}$ up to approximately $8 \mathrm{Td}$. Ps-induced NDC has been predicted for various other gaseous systems including $\mathrm{Ar}, \mathrm{H}_{2}$, and $\mathrm{H}_{2} \mathrm{O} .{ }^{48-50}$ The mechanism is well understood-as the reduced field increases, the modification to the time rate of change of the center of mass of the positron swarm, brought about by preferential loss of positrons (to positronium) at the front of the swarm, increases at a greater rate than the advective component, and hence, NDC in the bulk then emerges. Similar qualitative behavior is present for positrons in Mg; however, this NDC effect is greatly magnified for positrons in $\mathrm{Mg}$ due to the increased magnitude of the associated cross section. The NDC effect is more pronounced here with the bulk drift velocity reducing by over an order of magnitude, with the region for NDC occurring over a much larger range of reduced fields.

\subsection{Comparison of electron and positron transport in gaseous $\mathrm{Be}$ and $\mathrm{Mg}$}

While the scattering channels common to positrons and electrons have distinctly different energy dependencies of their cross sections [the exception to this is for direct ionization scattering where for each species (i.e., either Be or $\mathrm{Mg}$ ) and above about $100 \mathrm{eV}$, both our OP and ROP results suggest their cross sections are largely identical], which can be manifestly expressed macroscopically in the field dependence of the transport coefficients, so too can the different processes available to positrons (e.g., positronium formation) over electrons lead to different transport behavior. In Fig. 10, we highlight some important observations on the differences between electron and positron transport in $\mathrm{Be}$ and $\mathrm{Mg}$ gases.

For Be gas, we observe that the electron drift velocity is approximately an order of magnitude greater than the positron drift velocity at very low $E / n_{0}$. Likewise, we observe that the electrons depart from the thermal velocity distribution prior to the positrons (i.e., departure from linear variation with $E / n_{0}$ ) with the mean energy 
TABLE 6. Recommended cross sections for positron scattering from Mg. The errors on the elastic ICS are $\pm 20 \% ; \pm 30 \%$ on the positronium formation ICS; an uncertainty of $\pm 40 \%$ on the sum of the discrete-excitation electronic-states (inelastic); $\pm 20 \%$ on the ionization ICS; and $\pm 20 \%$ on the TCS

\begin{tabular}{|c|c|c|c|c|c|}
\hline Energy (eV) & $\begin{array}{c}\text { Elastic ICS } \\
\left(\times 10^{-16} \mathrm{~cm}^{2}\right)\end{array}$ & $\begin{array}{l}\text { Ps formation } \\
\left(\times 10^{-16} \mathrm{~cm}^{2}\right)\end{array}$ & $\begin{array}{c}\text { Excitation } \\
\left(\times 10^{-16} \mathrm{~cm}^{2}\right)\end{array}$ & $\begin{array}{l}\text { Direct ionization ICS } \\
\quad\left(\times 10^{-16} \mathrm{~cm}^{2}\right)\end{array}$ & $\begin{array}{c}\text { Total } \\
\left(\times 10^{-16} \mathrm{~cm}^{2}\right)\end{array}$ \\
\hline $1.000000 \times 10^{-5}$ & 183.981 & 0.000000 & 0.000000 & 0.000000 & 183.981 \\
\hline $2.000000 \times 10^{-4}$ & 194.549 & 0.000000 & 0.000000 & 0.000000 & 194.549 \\
\hline $1.000000 \times 10^{-3}$ & 209.769 & 0.000000 & 0.000000 & 0.000000 & 209.769 \\
\hline $1.000000 \times 10^{-2}$ & 264.665 & 0.000000 & 0.000000 & 0.000000 & 264.665 \\
\hline $5.000000 \times 10^{-2}$ & 336.342 & 0.000000 & 0.000000 & 0.000000 & 336.342 \\
\hline $7.000000 \times 10^{-2}$ & 409.715 & 0.000000 & 0.000000 & 0.000000 & 409.715 \\
\hline 0.140000 & 810.071 & 0.000000 & 0.000000 & 0.000000 & 810.071 \\
\hline 0.170000 & 888.269 & 0.000000 & 0.000000 & 0.000000 & 888.269 \\
\hline 0.210000 & 845.020 & 0.000000 & 0.000000 & 0.000000 & 845.020 \\
\hline 0.330000 & 560.358 & 0.000000 & 0.000000 & 0.000000 & 560.358 \\
\hline 0.450000 & 414.207 & 0.000000 & 0.000000 & 0.000000 & 414.207 \\
\hline 0.700000 & 288.537 & 0.000000 & 0.000000 & 0.000000 & 288.537 \\
\hline 0.875000 & 255.605 & 0.617463 & 0.000000 & 0.000000 & 256.222 \\
\hline 0.900000 & 251.858 & 1.33687 & 0.000000 & 0.000000 & 253.195 \\
\hline 0.950000 & 249.199 & 3.02818 & 0.000000 & 0.000000 & 252.228 \\
\hline 1.00000 & 246.541 & 11.1265 & 0.000000 & 0.000000 & 257.667 \\
\hline 1.10000 & 236.500 & 19.2430 & 0.000000 & 0.000000 & 255.743 \\
\hline 1.20000 & 221.549 & 24.3536 & 0.000000 & 0.000000 & 245.903 \\
\hline 1.70000 & 157.160 & 33.3589 & 0.000000 & 0.000000 & 190.519 \\
\hline 2.00000 & 135.756 & 36.6287 & 0.000000 & 0.000000 & 172.385 \\
\hline 2.80000 & 102.059 & 40.0462 & $6.780000 \times 10^{-2}$ & 0.000000 & 142.173 \\
\hline 2.90000 & 98.8625 & 40.0854 & $9.550000 \times 10^{-2}$ & 0.000000 & 139.043 \\
\hline 3.20000 & 91.0475 & 39.8660 & 0.142000 & 0.000000 & 131.056 \\
\hline 3.80000 & 78.7134 & 38.9059 & 0.179600 & 0.000000 & 117.799 \\
\hline 4.20000 & 71.9952 & 38.1249 & 0.190200 & 0.000000 & 110.310 \\
\hline 4.40000 & 68.8814 & 37.1417 & 2.58810 & 0.000000 & 108.611 \\
\hline 4.60000 & 65.9575 & 36.2306 & 4.69740 & 0.000000 & 106.885 \\
\hline 5.20000 & 58.0963 & 33.9728 & 8.82940 & 0.000000 & 100.898 \\
\hline 6.40000 & 45.2680 & 29.8119 & 14.2539 & 0.000000 & 89.3338 \\
\hline 7.80000 & 33.9246 & 25.1979 & 17.9762 & 0.169973 & 77.2687 \\
\hline 8.00000 & 32.5581 & 24.5069 & 18.3502 & 0.566464 & 75.9816 \\
\hline 8.60000 & 29.0637 & 22.4717 & 19.1469 & 1.18134 & 71.8637 \\
\hline 11.0000 & 19.6689 & 17.3249 & 20.0040 & 3.14575 & 60.1436 \\
\hline 13.0000 & 15.6264 & 14.1717 & 20.4103 & 4.80101 & 55.0094 \\
\hline 15.0000 & 13.2930 & 11.7350 & 20.4161 & 6.34267 & 51.7868 \\
\hline 20.0000 & 9.06761 & 7.29118 & 19.2904 & 7.65800 & 43.3072 \\
\hline 25.0000 & 6.91139 & 4.82663 & 18.3960 & 7.56110 & 37.6951 \\
\hline 30.0000 & 6.08586 & 3.55684 & 17.6104 & 7.01875 & 34.2718 \\
\hline 40.0000 & 5.20545 & 2.16770 & 15.8525 & 5.98839 & 29.2140 \\
\hline 50.0000 & 4.60955 & 1.32292 & 14.1125 & 5.23107 & 25.2760 \\
\hline 65.0000 & 3.73636 & 0.893427 & 12.0858 & 4.54869 & 21.2643 \\
\hline 85.0000 & 3.03568 & 0.572387 & 10.2050 & 3.90210 & 17.7152 \\
\hline 100.000 & 2.68026 & 0.422003 & 9.15150 & 3.53038 & 15.7842 \\
\hline 120.000 & 2.34214 & 0.278045 & 8.27100 & 3.19799 & 14.0892 \\
\hline 140.000 & 2.09508 & 0.174631 & 7.44770 & 2.89871 & 12.6161 \\
\hline 160.000 & 1.90590 & 0.102884 & 6.75630 & 2.65619 & 11.4213 \\
\hline 180.000 & 1.75555 & $5.213755 \times 10^{-2}$ & 6.16250 & 2.46437 & 10.4346 \\
\hline 190.000 & 1.69131 & $3.239550 \times 10^{-2}$ & 5.89550 & 2.37373 & 9.99294 \\
\hline 200.000 & 1.63299 & $1.556839 \times 10^{-2}$ & 5.64600 & 2.28608 & 9.58063 \\
\hline 300.000 & 1.24581 & 0.000000 & 3.95530 & 1.73832 & 6.93943 \\
\hline 400.000 & 1.03431 & 0.000000 & 2.91650 & 1.41786 & 5.36867 \\
\hline 500.000 & 0.897098 & 0.000000 & 2.26010 & 1.20831 & 4.36551 \\
\hline
\end{tabular}


TABLE 6. (Continued.)

\begin{tabular}{lcccc}
\hline \hline Energy $(\mathrm{eV})$ & $\begin{array}{c}\text { Elastic ICS } \\
\left(\times 10^{-16} \mathrm{~cm}^{2}\right)\end{array}$ & $\begin{array}{c}\text { Ps formation } \\
\left(\times 10^{-16} \mathrm{~cm}^{2}\right)\end{array}$ & $\begin{array}{c}\text { Excitation } \\
\left(\times 10^{-16} \mathrm{~cm}^{2}\right)\end{array}$ & $\begin{array}{c}\text { Direct ionization ICS } \\
\left(\times 10^{-16} \mathrm{~cm}^{2}\right)\end{array}$ \\
\hline 700.000 & 0.724155 & 0.000000 & 1.56740 & 0.947113 \\
$\left.1 \times 10^{-16} \mathrm{~cm}^{2}\right)$
\end{tabular}

(not shown) of the electrons initially increasing more rapidly with $E / n_{0}$ than the positrons. Both of these macroscopic observations reflect the substantially higher elastic MTCS for positrons over electrons for energies well below $0.1 \mathrm{eV}$. As the reduced field increases further, the mean energy of the positrons increases more rapidly than for electrons due to a combination of a falling positron elastic cross
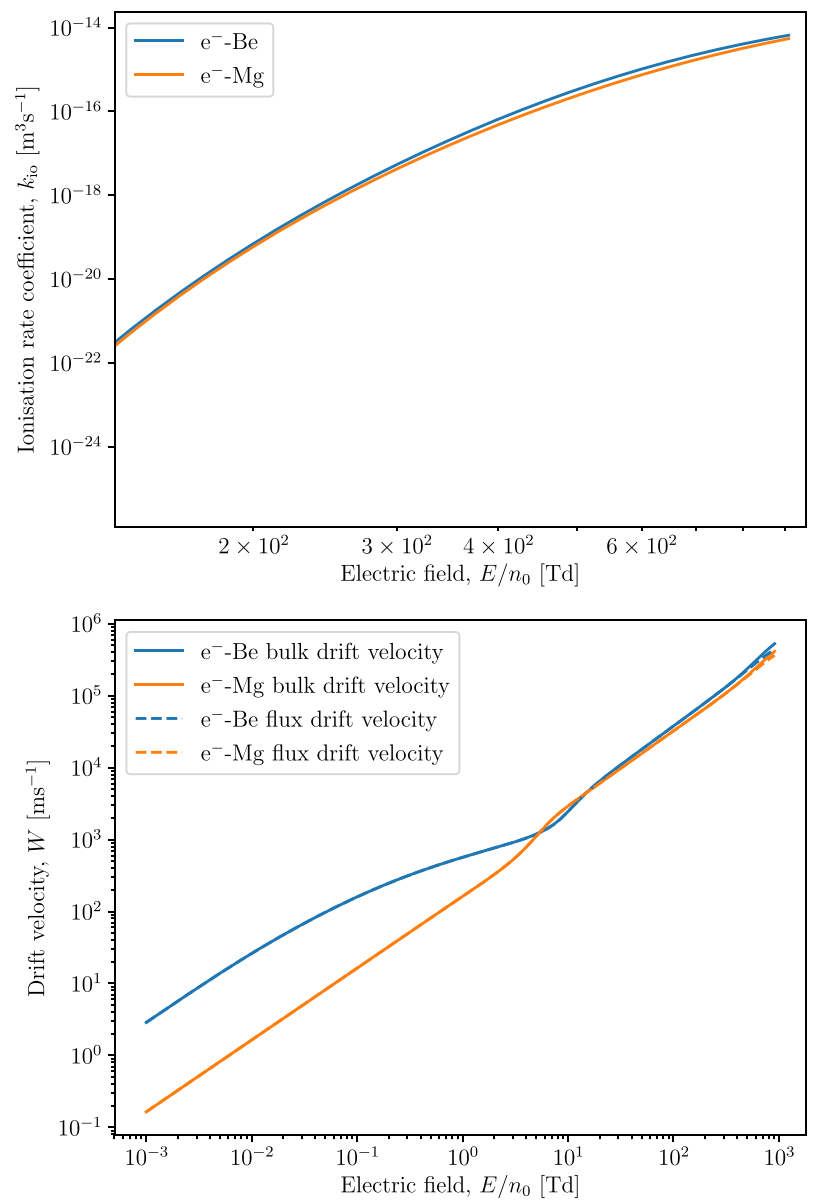

FIG. 8. Comparison of the calculated rate coefficients for ionization (top), bulk (solid), and flux (dashed) drift velocities (bottom) for electrons in $\mathrm{Be}$ (blue) and $\mathrm{Mg}$ (orange) vapor at $750 \mathrm{~K}$ over a range of reduced electric fields. See also the legend for further details. section and the distinct differences in the electronic excitation processes for electrons and positrons. For electrons in Be, the electronic excitation process is operative at lower energies than that for
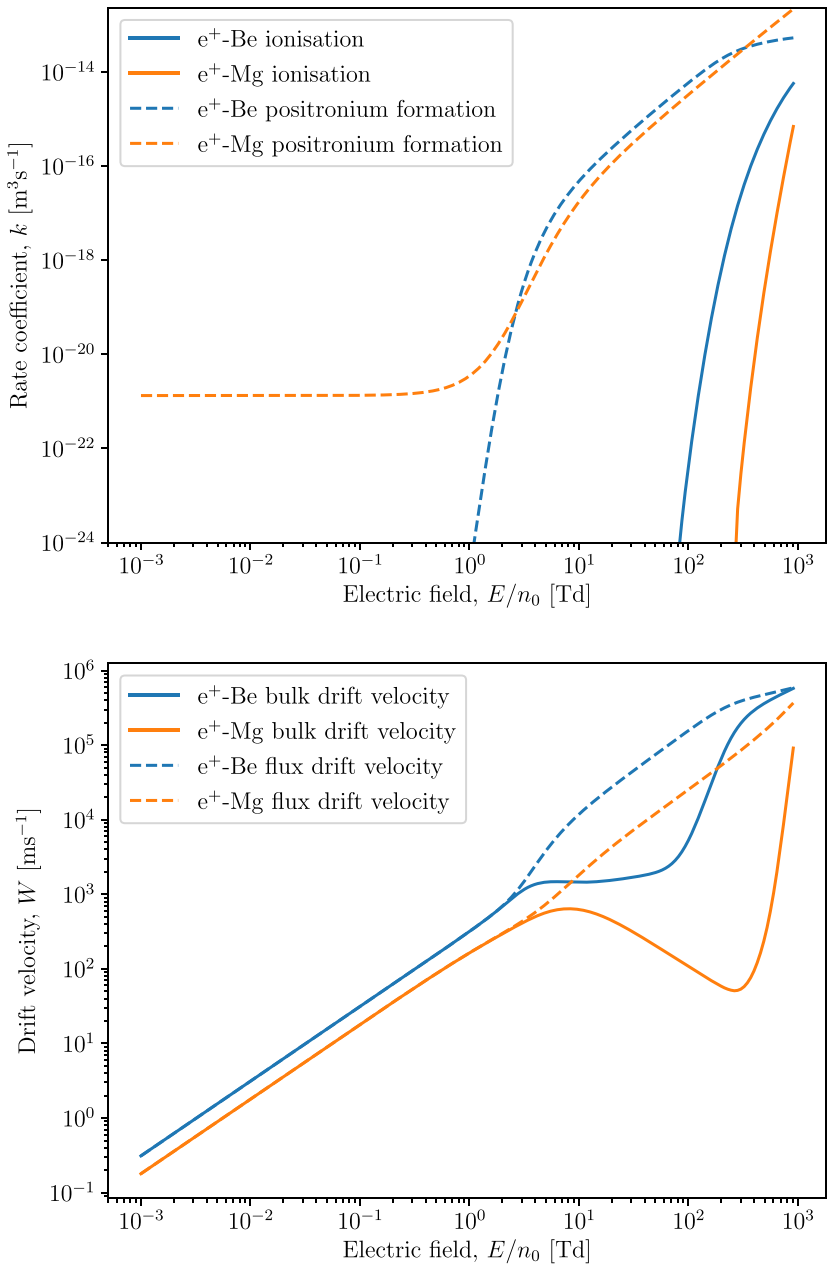

FIG. 9. Comparison of the calculated rate coefficients (top) for ionization (solid) and positronium formation (dashed), bulk (solid) and flux (dashed) drift velocities (bottom) for positrons in $\mathrm{Be}$ (blue) and $\mathrm{Mg}$ (orange) vapor at $750 \mathrm{~K}$ over a range of reduced electric fields. See also the legend for further details. 

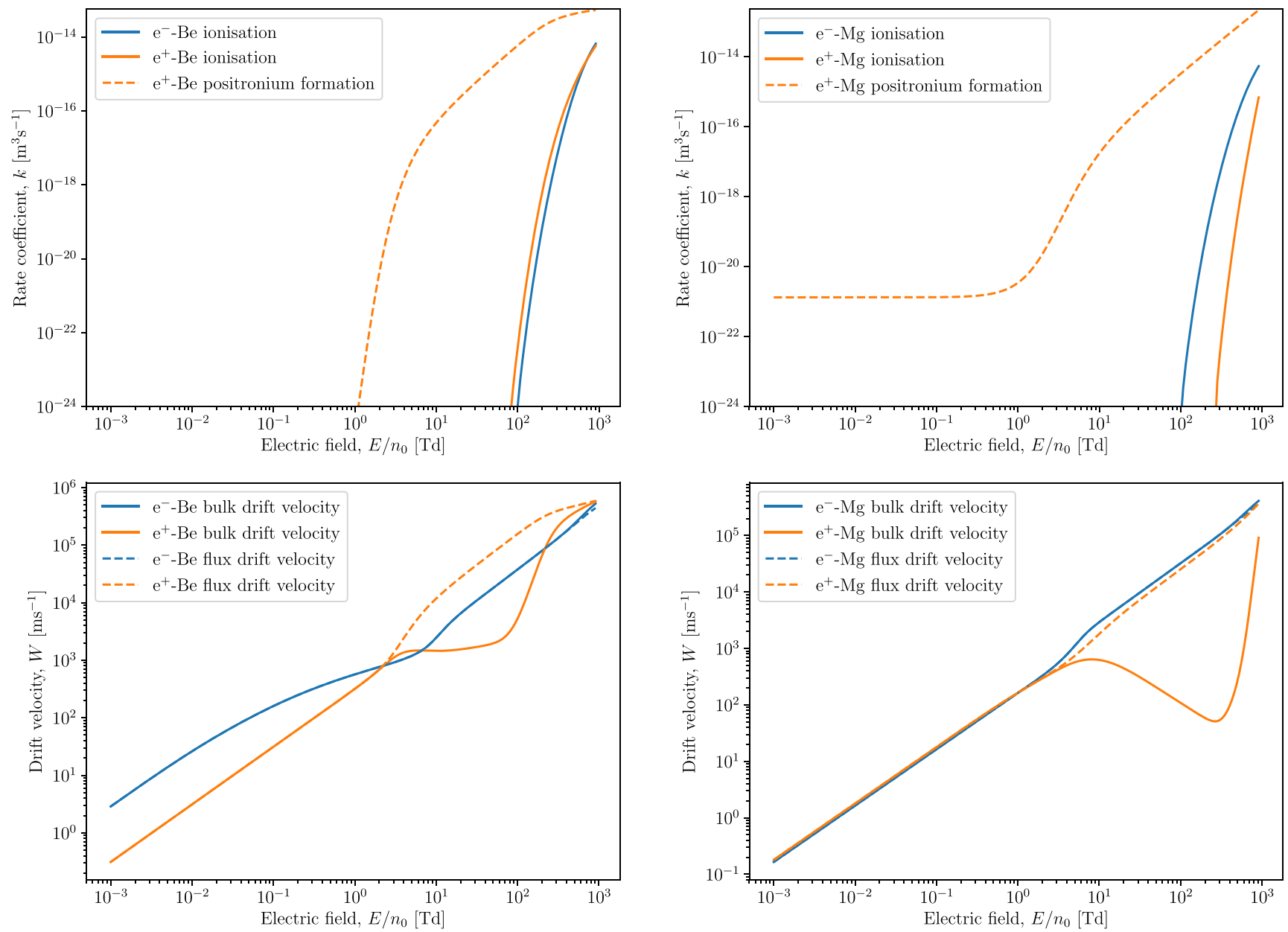

FIG. 10. Comparison of the calculated rate coefficients (top) for ionization (solid) and positronium formation (dashed), bulk (solid) and flux (dashed) drift velocities (bottom row) for electrons (blue) and positrons (orange) in $\mathrm{Be}$ (left column) and $\mathrm{Mg}$ (right column) vapor at $750 \mathrm{~K}$ over a range of reduced electric fields. See also the legend for further details.

positrons, in a nonrelativistic description, with the cross section for electrons being significantly higher in magnitude in the energy range from $\sim 2.6$ to $10 \mathrm{eV}$, above which they are very similar. This mode for absorbing energy from the field for electrons then results in the mean energy of the electrons rising more slowly with $E / n_{0}$ than for positrons. The distinctly different nature of the collisional processes is explicitly manifest in the modification to the center-ofmass (or bulk) drift velocity. As detailed above, the Ps-formation process results in a significant decrease in the bulk drift velocity in the region where this channel is significant. Positron-induced ionization does not impact upon the motion of the center of mass since it is a conservative process, in contrast to the case for electron-impact ionization, where the bulk drift velocity lies above the flux drift velocity where this collisional process is significant. For electrons and positrons in $\mathrm{Mg}$, we observe qualitatively similar behaviors though these are magnified given the strength of the Ps-formation process for positrons.

\section{Conclusions}

We have reported on results from original OP and ROP calculations for positron scattering from beryllium and magnesium. Together with the somewhat limited results from previous work, ${ }^{2}$ we were able to use our new computations to construct recommended databases for $e^{+}-\mathrm{Be}$ and $e^{+}-\mathrm{Mg}$ scattering. We have also presented a brief comparative study of the electron and positron transport in $\mathrm{Be}$ and $\mathrm{Mg}$ gases at $750 \mathrm{~K}$ and shown that the transport coefficients are typically distinctly different for the two metal vapors. These differences represent the macroscopic manifestation of differences in the electron and positron cross sections for scattering processes they have in common and, also more importantly, differences in the fundamental nature of the processes available to electrons and positrons. The latter is strikingly highlighted in the effect of the Ps-formation channel which is predicted to generate NDC for positrons in both $\mathrm{Be}$ and $\mathrm{Mg}$, yet is predicted to be absent for electrons in both gases. Also, we should highlight that experimental transport measurements for 
both these species are highly desirable as they might represent the best opportunity to validate the low-energy cross sections $(<0.1 \mathrm{eV})$ and importantly resonances in these cross sections. This highlights the importance of an accurate and complete set of cross sections for electrons and positrons when modeling their transport in metal vapors, and indeed, the proposed transport measurements also represent an independent method of testing the accuracy and selfconsistency of the cross section sets.

\section{Acknowledgments}

This work was financially supported, in part, by the Spanish Ministerio de Ciencia, Innovation y Universidades (Project No. FIS2016-80440) and the Australian Research Council (Project Nos. DP160102787, DP180101655, and DP190100696). We thank Dr. L. Campbell for his help with some aspects of this study. Finally, we also thank Professor I. Bray for providing us tables of his CCC data and Dr. M. Bromley for providing us with tables of his results.

\section{References}

${ }^{1}$ L. Chiari and A. Zecca, Eur. Phys. J. D 68, 297 (2014).

${ }^{2}$ K. Ratnavelu, M. J. Brunger, and S. J. Buckman, J. Phys. Chem. Ref. Data 48, 023102 (2019).

${ }^{3}$ M. W. J. Bromley, J. Mitroy, and G. Ryzhikh, J. Phys. B: At., Mol. Opt. Phys. 31, 4449 (1998).

${ }^{4}$ J. Mitroy and I. A. Ivanov, Phys. Rev. A 65, 042705 (2002).

${ }^{5}$ D. D. Reid and J. M. Wadehra, J. Phys. B: At., Mol. Opt. Phys. 47, 225211 (2014).

${ }^{6}$ L. A. Poveda, D. Assafrão, and J. R. Mohallem, Eur. Phys. J. D 70, 152 (2016).

${ }^{7}$ T. S. Stein, M. Horte, J. Jiang, W. E. Kaupplia, C. K. Kwan, H. Li, and S. Zhou, Nucl. Instrum. Methods B 143, 68 (1998).

${ }^{8}$ T. S. Stein, J. Jiang, W. E. Kauppila, C. K. Kwan, H. Li, E. Surdutovich, and S. Zhou, Can. J. Phys. 74, 313 (1996).

${ }^{9}$ E. Surdutovich, M. Horte, W. E. Kauppila, C. K. Kwan, and T. S. Stein, Phys. Rev. A 68, 022709 (2003).

${ }^{10}$ J. Mitroy, J. Y. Zhang, M. W. J. Bromley, and S. I. Young, Phys. Rev. A 78, 012715 (2008).

${ }^{11}$ J. S. Savage, D. V. Fursa, and I. Bray, Phys. Rev. A 83, 062709 (2011).

${ }^{12}$ R. Utamuratov, D. V. Fursa, A. S. Kadyrov, A. V. Lugovskoy, J. S. Savage, and I. Bray, Phys. Rev. A 86, 062702 (2012).

${ }^{13}$ G. F. Gribakin and W. A. King, Can. J. Phys. 74, 449 (1996).

${ }^{14}$ R. N. Hewitt, C. Noble, B. H. Bransdon, and C. J. Joachain, Can. J. Phys. 74, 559 (1996).

${ }^{15}$ C. Chang and Y. Zhou, Phys. Rev. A 73, 024701 (2006).

${ }^{16}$ C. Makochekanwa, M. Hoshino, H. Kato, O. Sueoka, M. Kimura, and H. Tanaka, Phys. Rev. A 77, 042717 (2008).

${ }^{17}$ O. Sueoka, C. Makochekanwa, H. Tanina, and M. Kimura, Phys. Rev. A 72, 042705 (2005).

${ }^{18}$ L. Chiari, A. Zecca, F. Blanco, G. García, and M. J. Brunger, Phys. Rev. A 91, 012711 (2015).

${ }^{19}$ L. Chiari, A. Zecca, G. García, F. Blanco, and M. J. Brunger, J. Phys. B: At., Mol. Opt. Phys. 46, 235202 (2013).

${ }^{20}$ A. Zecca, C. Perazzolli, and M. J. Brunger, J. Phys. B: At., Mol. Opt. Phys. 38, 2079 (2005).

${ }^{21}$ M. J. Brunger, S. J. Buckman, and K. Ratnavelu, J. Phys. Chem. Ref. Data 46, 023102 (2017).
${ }^{22}$ R. P. McEachran, F. Blanco, G. García, and M. J. Brunger, J. Phys. Chem. Ref. Data 47, 033103 (2018).

${ }^{23}$ R. P. McEachran, F. Blanco, G. García, P. W. Stokes, R. D. White, and M. J. Brunger, J. Phys. Chem. Ref. Data 47, 043104 (2018).

${ }^{24}$ D. D. Reid and J. M. Wadehra, J. Phys. B: At., Mol. Opt. Phys. 29, L127 (1996).

${ }^{25}$ W. Tattersall, L. Chiari, J. R. Machacek, E. Anderson, R. D. White, M. J. Brunger, S. J. Buckman, G. García, F. Blanco, and J. P. Sullivan, J. Chem. Phys. 140, 044320 (2014).

${ }^{26}$ L. Chiari, A. Zecca, S. Girardi, E. Trainotti, G. García, F. Blanco, R. P. McEachran, and M. J. Brunger, J. Phys. B: At., Mol. Opt. Phys. 45, 215206 (2012).

${ }^{27}$ R. P. McEachran, D. L. Morgan, A. G. Ryman, and A. D. Stauffer, J. Phys. B: At., Mol. Opt. Phys. 10, 663 (1977).

${ }^{28}$ A. C. L. Jones, C. Makochekanwa, P. Caradonna, D. S. Slaughter, J. R. Machacek, R. P. McEachran, J. P. Sullivan, S. J. Buckman, A. D. Stauffer, I. Bray, and D. V. Fursa, Phys. Rev. A 83, 032701 (2011).

${ }^{29}$ F. Blanco, A. M. Roldán, K. Krupa, R. P. McEachran, R. D. White, S. Marjanović, Z. Lj. Petrović, M. J. Brunger, J. R. Machacek, S. J. Buckman, J. P. Sullivan, L. Chiari, P. Limão-Vieira, and G. García, J. Phys. B: At., Mol. Opt. Phys. 49, 145001 (2016), ${ }^{30}$ S. Chen, R. P. McEachran, and A. D. Stauffer, J. Phys. B: At., Mol. Opt. Phys. 41, 025201 (2008).

${ }^{31}$ K. Bartschat, R. P. McEachran, and A. D. Stauffer, J. Phys. B: At., Mol. Opt. Phys. 21, 2789 (1988).

${ }^{32}$ K. Bartschat, R. P. McEachran, and A. D. Stauffer, J. Phys. B: At., Mol. Opt. Phys. 23, 2349 (1990).

${ }^{33}$ R. P. McEachran, D. L. Morgan, A. G. Ryman, and A. D. Stauffer, J. Phys. B: At., Mol. Opt. Phys. 11, 951 (1978).

${ }^{34}$ R. P. McEachran and A. D. Stauffer, J. Phys. B: At., Mol. Opt. Phys. 46, 075203 (2013).

${ }^{35}$ D. D. Reid and J. M. Wadehra, J. Phys. B: At., Mol. Opt. Phys. 30, 2318 (1997).

${ }^{36}$ D. J. Murtagh, M. Szluinska, J. Moxom, P. Van Reeth, and G. Laricchia, J. Phys. B: At., Mol. Opt. Phys. 38, 3857 (2005).

${ }^{37}$ Y. Itikawa, J. Phys. Chem. Ref. Data 46, 043103 (2017).

${ }^{38}$ Y. Itikawa, J. Phys. Chem. Ref. Data 45, 033106 (2016).

${ }^{39}$ R. P. McEachran and A. D. Stauffer, J. Phys. B 52, 115203 (2019).

${ }^{40}$ P. Caradonna, A. Jones, C. Makochekanwa, D. S. Slaughter, J. P. Sullivan, S. J. Buckman, I. Bray, and D. V. Fursa, Phys. Rev. A 80, 032710 (2009).

${ }^{41}$ A. Zecca, L. Chiari, E. Trainotti, D. V. Fursa, I. Bray, and M. J. Brunger, Eur. Phys. J. D 64, 317 (2011)

${ }^{42}$ A. Zecca, L. Chiari, E. Trainotti, D. V. Fursa, I. Bray, A. Sarkar, S. Chattopadhyay, K. Ratnavelu, and M. J. Brunger, J. Phys. B: At., Mol. Opt. Phys. 45, 015203 (2012).

${ }^{43}$ A. Zecca, L. Chiari, E. Trainotti, and M. J. Brunger, J. Phys. B: At., Mol. Opt. Phys. 45, 085203 (2012).

${ }^{44}$ R. D. White, D. Cocks, G. Boyle, M. Casey, N. Garland, D. Konovalov, B. Philippa, P. Stokes, J. de Urquijo, O. González-Magaña, R. P. McEachran, S. J. Buckman, M. J. Brunger, G. Garcia, S. Dujko, and Z. Lj. Petrovic, Plasma Sources Sci. Technol. 27, 053001 (2018).

${ }^{45}$ G. J. Boyle, W. J. Tattersall, D. G. Cocks, R. P. McEachran, and R. D. White, Plasma Sources Sci. Technol. 26, 024007 (2017).

${ }^{46}$ R. D. White, R. E. Robson, B. Schmidt, and M. A. Morrison, J. Phys. D: Appl. Phys. 36, 3125 (2003).

${ }^{47}$ G. J. Boyle, W. J. Tattersall, D. G. Cocks, S. Dujko, and R. D. White, Phys. Rev. A 91, 052710 (2015).

${ }^{48}$ A. Banković, S. Dujko, R. D. White, J. P. Marler, S. J. Buckman, S. Marjanović, G. Malović, G. García, and Z. Lj. Petrović, New J. Phys. 14, 035003 (2012).

${ }^{49}$ A. Banković, S. Dujko, R. D. White, S. J. Buckman, and Z. Lj. Petrović, Nucl. Instrum. Methods B 279, 92 (2012).

${ }^{50}$ M. Šuvakov, Z. L. Petrović, J. P. Marler, S. J. Buckman, R. E. Robson, and G. Malović, New J. Phys. 10, 053034 (2008). 